\title{
On the Value of Correlation
}

\section{Itai Ashlagi}

Harvard Business School,

Harvard University,

Boston, MA, 02163,USA

\section{Dov Monderer}

Faculty of Industrial Engineering and Management, Technion - Israel Institute of Technology,

Haifa 32000, Israel

Moshe Tennenholtz

Microsoft Israel REDD Center, 13 Shenkar St., Herzeliya 46725, Israel, and

Faculty of Industrial Engineering and Management,

Technion - Israel Institute of Technology,

Haifa 32000, Israel
IASHLAGI@HBS.EDU

DOV@IE.TECHNION.AC.IL

MOSHET@MICROSOFT.COM

\begin{abstract}
Correlated equilibrium generalizes Nash equilibrium to allow correlation devices. Correlated equilibrium captures the idea that in many systems there exists a trusted administrator who can recommend behavior to a set of agents, but can not enforce such behavior. This makes this solution concept most appropriate to the study of multi-agent systems in AI. Aumann showed an example of a game, and of a correlated equilibrium in this game in which the agents' welfare (expected sum of players' utilities) is greater than their welfare in all mixed-strategy equilibria. Following the idea initiated by the price of anarchy literature this suggests the study of two major measures for the value of correlation in a game with nonnegative payoffs:
\end{abstract}

1. The ratio between the maximal welfare obtained in a correlated equilibrium to the maximal welfare obtained in a mixed-strategy equilibrium. We refer to this ratio as the mediation value.

2. The ratio between the maximal welfare to the maximal welfare obtained in a correlated equilibrium. We refer to this ratio as the enforcement value.

In this work we initiate the study of the mediation and enforcement values, providing several general results on the value of correlation as captured by these concepts. We also present a set of results for the more specialized case of congestion games, a class of games that received a lot of attention in the recent literature.

\section{Introduction}

Much work in the area of multi-agent systems adopts game-theoretic reasoning. This is due to the fact that many existing systems consist of self-motivated participants, each of which attempts to optimize his own performance. As a result the Nash equilibrium, the central solution concept in game theory, has become a major tool in the study and analysis of multi-agent systems. Nash equilibrium captures multi-agent behavior which is stable against 
unilateral deviations. Naturally, a system that is fully controlled by a designer can enforce behaviors which lead to a higher welfare than the one obtained in a fully decentralized system in which agents behave selfishly and follow some Nash equilibrium. The comparison between these quantities is studied under the title of work on "the price of anarchy" (Koutsoupias \& Papadimitriou, 1999; Roughgarden \& Tardos, 2002; Christodoulou \& Koutsoupias, 2005), and is a subject of much interest in computer science. However, fully controlled systems versus fully uncontrolled systems are two extreme points. As was acknowledged in various works in AI (Shoham \& Tennenholtz, 1995a, 1995b) one of the main practical approaches to dealing with realistic systems is to consider systems with some limited centralized control. Indeed, in most realistic systems there is a designer who can recommend behavior; this should be distinguished from the strong requirement that the designer can dictate behavior. Correlated equilibrium, introduced by Aumann (1974), is the most famous game-theoretic solution concept referring to a designer who can recommend but not enforce behavior. In a game in strategic form, a correlated strategy is a probability distribution over the set of strategy profiles, where a strategy profile is a vector of strategies, one for each player. A correlated strategy is utilized as follows: A strategy profile is selected according to the distribution, and every player is informed about her strategy in the profile. This selected strategy for the player is interpreted as a recommendation of play. Correlated strategies are most natural, since they capture the idea of a system administrator/reliable party who can recommend behavior but can not enforce it. Hence, correlated strategies make perfect sense in the context of congestion control, load balancing, trading, etc. A correlated strategy is called a correlated equilibrium if it is better off for every player to obey her recommended strategy if she believes that all other players obey their recommended strategies ${ }^{1}$. Correlated equilibrium makes perfect sense in the context of work on multi-agent systems in $\mathrm{AI}$ in which there exists a mediator who can recommend behavior to the agents. ${ }^{2}$ A major potential benefit of a mediator who is using a correlated equilibrium is to attempt to improve the welfare of selfish players. In this paper, the welfare obtained in a correlated strategy is defined to be the expected sum of the utilities of the players, and it is referred to as the welfare obtained in this correlated strategy.

A striking example introduced in Aumann's seminal paper (1974) is of a two-player two-strategy game, where the welfare obtained in a correlated equilibrium is higher than the welfare obtained in every mixed-strategy equilibrium of the game. A modification of Aumann's example serves us as a motivating example.

\section{Aumann's Example:}

1. Every correlated strategy defines a Bayesian game in which the private signal of every player is her recommended strategy. It is a correlated equilibrium if obeying the recommended strategy by every player is a pure-strategy equilibrium in this Bayesian game.

2. The use of mediators in obtaining desired behaviors, in addition to improving social welfare, has been further studied, (e.g., Monderer \& Tennenholtz, 2004, 2006; Rozenfeld \& Tennenholtz, 2007; Ashlagi, Monderer, \& Tennenholtz, 2008). However, the mediators discussed in that work makes use of more powerful capabilities than just making recommendation based on probabilistic coin flips. 


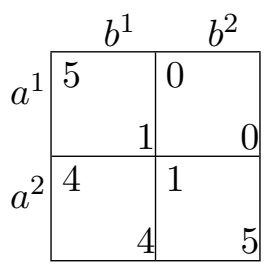

As a result, Aumann's example suggests that correlation may be a way to improve welfare while still assuming that players are rational in the classical game-theoretic sense. ${ }^{3}$

In this game, there are three mixed-strategy equilibrium profiles. Two of them are obtained with pure strategies, $\left(a^{1}, b^{1}\right)$, and $\left(a^{2}, b^{2}\right)$. The welfare in each of these purestrategy equilibrium profiles equals six. There is an additional mixed-strategy equilibrium in which every player chooses each of her strategies with equal probabilities. The welfare obtained in this profile equals $5\left(=\frac{1}{4}(6+0+8+6)\right)$ because every entry in the matrix is played with probability $\frac{1}{4}$. Hence, the maximal welfare in a mixed-strategy equilibrium equals 6 . Consider the following correlated strategy: a probability of $1 / 3$ is assigned to every pure strategy profile but $\left(a^{1}, b^{2}\right)$. This correlated strategy is a correlated equilibrium. Indeed, when the row player is recommended to play $a^{1}$ she knows that the other player is recommended to play $b^{1}$, and therefore she strictly prefers to play $a^{1}$. When the row player is recommended to play $a^{2}$ the conditional probability of each of the columns is half, and therefore she weakly prefers to play $a^{2}$. Similar argument applied to the column player shows that the correlated strategy is indeed a correlated equilibrium. The welfare associated with this correlated equilibrium equals $\frac{20}{3}\left(=\frac{1}{3}(6+8+6)\right)$.

The above discussion suggests one may wish to consider the value of correlation in games. In order to address the challenge of studying the value of correlation, we tackle two fundamental issues:

- How much can the society/system gain by adding a correlation device, where we assume that without such a device the agents play a mixed-strategy equilibrium.

- How much does the society/system loose from the fact that the correlation device can only recommend (and can not enforce) a course of action?

Accordingly we introduce two measures, the mediation value and the enforcement value. These measures make sense mainly for games with nonnegative utilities, which are the focus of this paper.

The mediation value measures the ratio between the maximal welfare in a correlated equilibrium to the maximal welfare in a mixed-strategy equilibrium. Notice that the higher the mediation value is, the more correlation helps. Hence, the mediation value measures the value of a reliable mediator who can just recommend a play in a model in which there is an anarchy without the mediator, where anarchy is defined to be the situation in which the players use the welfare-best mixed-strategy equilibrium, that is, anarchy is the best outcome reached by rational and selfish agents. ${ }^{4}$

3. Other advantages are purely computational ones. As has been recently shown, correlated equilibrium can be computed in polynomial time even for structured representations of games (Kakade, Kearns, Langford, \& Ortiz, 2003; Papadimitriou, 2005).

4. The phenomenon of multiple equilibria forces a modeling choice of the concept of anarchy, which could have been defined also as the welfare-worst mixed-strategy equilibrium, or as a convex combination of 
In Aumman's example it can be shown that the correlated equilibrium introduced above is the best correlated equilibrium, i.e., it attains the maximal welfare among all correlated equilibria in the game. Hence, the mediation value of Aumann's example is $\frac{10}{9}$.

The enforcement value measures the ratio between the maximal welfare to the maximal welfare in a correlated equilibrium. That is, it is the value of a center who can dictate a course of play with respect to a mediator who can just use correlation devices in equilibrium. As the maximal welfare in Aumann's example is 8, the enforcement value in this game equals $\frac{6}{5}$.

In this paper we establish general and basic results concerning the measures defined above. We consider the mediation (enforcement) value of classes of games, which is defined to be the least upper bound of the mediation (enforcement) values of the games in the class. We first study general games. Then we consider the important class of congestion games (Rosenthal, 1973; Monderer \& Shapley, 1996). Indeed, this class of games is perhaps the most applicable to the game theory and CS synergy. In particular, results regarding the price of anarchy have been obtained for congestion games. We restrict our study to simple congestion games.

Next we summarize our main results and discuss some related literature.

\subsection{Main Results for General Games}

Aumann's example implies that the mediation value of the class of two-player two-strategy $(2 \times 2)$ games is at least $10 / 9$. In this paper it is proved that the mediation value of this class equals $4 / 3$. Next, more complex games are studied. In particular we consider two possible minimal extensions of $2 \times 2$ games: Two-player games with three strategies for one of the players and two strategies for the other, and three-player games with two strategies for each player. It is shown that the mediation value of each of these classes is unbounded, i.e., it equals $\infty$. Consequently, the mediation value is unbounded for classes of larger games. ${ }^{5}$ This should be interpreted as a positive result, showing the extreme power of correlation.

Considering the enforcement value, it is proved that it equals $\infty$ for the classes of $2 \times 2$ or larger games. The proof of this result uses games with weakly dominant strategies. We show, however, that the enforcement value of the class of three-player two-strategy games without weakly dominated strategies also equals $\infty$.

\subsection{Main Results for Simple Congestion Games}

In a simple congestion game there is a set of facilities. Every facility $j$ is associated with a nonnegative payoff function $w_{j}$. Every player chooses a facility, say facility $j$, and receives $w_{j}(k)$, where $k$ is the number of players that chose facility $j$.

For completeness we first deal with the simple case, in which there exist only two players, and show that the mediation value of the class of simple congestion games with two facilities equals $4 / 3$. In the more general case, in which there are $m \geq 2$ facilities, it is proved that the mediation value is bounded from above by 2 . However, it is proved that the mediation

the best-welfare and worst-welfare mixed strategy equilibrium .This choice is a matter of taste, and we chose the "best" option.

5. A game $\tilde{\Gamma}$ is larger than the game $\Gamma$ if it is obtained from $\Gamma$ by adding players, and/or by adding strategies to the players in $\Gamma$. 
value equals 1 for the class of simple congestion games with $n$ on-increasing facility payoff functions.

For the case of more than two players, we show that the mediation value is unbounded for the class of games with three players and two facilities with non-increasing payoffs. In contrast, in the linear case, it is proved that the mediation value is bounded from above by $\frac{\sqrt{5}+1}{2} \approx 1.618$ for the class of games with any number of players and two facilities with non-increasing linear payoff functions. We give an example for a game in this class whose mediation value equals $9 / 8$, leaving open a significant gap.

Additional special theorems are proved for simple congestion games with symmetric (identical) facilities; It is proved that, for every $n \geq 4$, the mediation value is higher than 1 for the class of two symmetric (identical) facilities with non-increasing payoffs and $n$ players. This further illustrates the power of correlation. Nevertheless, we show that every simple congestion game with any number of players and any number of symmetric facilities, in which the facility payoff functions satisfy a certain concavity requirement, the best mixedstrategy equilibrium obtains the maximal welfare, and therefore both the mediation value and the enforcement value of such a game equal 1.

Finally, we study the enforcement value in the case, in which there exist $n$ players and $m$ symmetric facilities with arbitrary facility payoff functions. We characterize the set of such games for which the enforcement value equals 1 , and as a result, determine the situations where correlation allows obtaining maximal welfare.

\subsection{Related Literature}

We end this introduction with a discussion of some relevant issues in the price of anarchy literature, and their potential relationships to the concepts of mediation value and enforcement value. ${ }^{6}$ In many situations it is natural to deal with nonnegative costs rather than utilities; indeed, the literature on the price of anarchy focused on such models. When translating the definition of price of anarchy ${ }^{7}$ to games with utilities and not with costs, the price of anarchy with utilities is the ratio between the maximal welfare to the minimal welfare obtained in a mixed-strategy equilibrium. The higher this number is, the value of a center is higher, where a center can enforce a course of play. Hence, the price of anarchy with utilities measures the value of a center with respect to anarchy, where a center can dictate a play, and when anarchy is measured by the worst social outcome reached by rational and selfish agents. Recently, Anshelevich, Dasgupta, Kleinberg, Tardos, Wexler, and Roughgarden (2004) defined price of stability in models with costs. ${ }^{8}$ Accordingly, the price of stability with utilities, is the ratio between the maximal welfare and the maximal welfare in a mixed-strategy equilibrium. A relevant concept using correlated equilibrium in models with costs has been defined independently by Christodoulou and Koutsoupias (2005), and is referred to as the price of stability of correlated equilibria. ${ }^{9}$ When translated to a model

6. The concept of the price of anarchy has received much attention in the recent computer science literature, (e.g., Marvonicolas \& Spirakis, 2001; Czumaj \& Vocking, 2002; Roughgarden, 2002; Roughgarden \& Tardos, 2002).

7. The price of anarchy is defined for games with costs as the ratio between the maximal cost in a mixedstrategy equilibrium to the minimal cost.

8. It is the ratio between the minimal cost in a mixed strategy equilibrium to the minimal cost.

9. It is the ratio between the minimal cost in a correlated equilibrium to the minimal cost. 
with utilities, the price of stability of correlated equilibria with utilities is the ratio between the maximal welfare and the maximal welfare in a mixed-strategy equilibrium, that is it is the enforcement value. ${ }^{10}$ However, results proved for one of the ratios in one of the models cannot be translated to results on the analogous ratio in the other model. This is due the fact that when moving from one model to the other does not only require multiplication by a negative constant, e.g. -1 , but the numbers also need to be shifted to remain nonnegative; needless to say, the corresponding ratios can be significantly changed by such shifting. ${ }^{11}$ We return to this discussion in Section 4.1.

\section{Basic Definitions}

A finite game in strategic form is a tuple $\Gamma=\left(N,\left(S^{i}\right)_{i \in N},\left(u^{i}\right)_{i \in N}\right) ; N$ is a nonempty finite set of players. Unless otherwise specified we assume that $N=\{1,2, \ldots, n\}, \quad n \geq 1$. For each $i \in N, S^{i}$ is a finite set of strategies of player $i$. Let $\mathbf{S}=S^{1} \times S^{2} \times \cdots \times S^{n}$ be the set of strategy profiles (n-tuples). An element of $\mathbf{S}$ is $\mathbf{s}=\left(s^{i}\right)_{i \in N}$. For each $i \in N$ and $\mathbf{s} \in \mathbf{S}$ let $\mathbf{s}^{-i}=\left(s^{1}, \ldots, s^{i-1}, s^{i+1}, \ldots s^{n}\right)$ denote the strategies played by everyone but $i$. Thus $\mathbf{s}=\left(\mathbf{s}^{-\mathbf{i}}, \mathbf{s}^{\mathbf{i}}\right)$. For each player $i \in N$, let $u^{i}: \mathbf{S} \rightarrow \mathbb{R}$ be the utility function of player $i . u^{i}(\mathbf{s})$ is the utility of player $i$ when the profile of strategies $\mathbf{s}$ is played. $\Gamma$ is called a nonnegative game if all utilities to all players are nonnegative, i.e., $u^{i}: \mathbf{S} \rightarrow \mathbb{R}_{+}$for every player $i$.

A player can also randomize among her strategies by using a mixed strategy - a distribution over her set of strategies. For any finite set $C, \Delta(C)$ denotes the set of probability distributions over $C$. Thus $P^{i}=\Delta\left(S^{i}\right)$ is the set of mixed strategies of player $i$. Let $p^{i} \in P^{i}$ be a mixed strategy of $i$. For every $s^{i} \in S^{i}, p^{i}\left(s^{i}\right)$ is the probability that player $i$ plays strategy $s^{i}$ in $p^{i}$. Every pure strategy $s^{i} \in S^{i}$ is, with the natural identification, a mixed strategy $p_{s^{i}} \in P^{i}$ in which

$$
p_{s^{i}}\left(t^{i}\right)=\left\{\begin{array}{cc}
1 & t^{i}=s^{i} \\
0 & t^{i} \neq s^{i}
\end{array}\right.
$$

$p_{s^{i}}$ is called a pure strategy, and $s^{i}$ is interchangeably called a strategy and a pure strategy (when it is identified with $p_{s^{i}}$ ). Let $\mathbf{P}=P^{1} \times P^{2} \times \cdots \times P^{n}$ be the set of mixed strategy profiles.

Let $s^{i}, t^{i} \in S^{i}$ be pure strategies of player $i$. We say that $s^{i}$ weakly dominates $t^{i}$, and $t^{i}$ is weakly dominated by $s^{i}$ if for all $\mathbf{s}^{-i} \in \mathbf{S}^{-\mathbf{i}}$

$$
u^{i}\left(s^{i}, \mathbf{s}^{-i}\right) \geq u^{i}\left(t^{i}, \mathbf{s}^{-i}\right),
$$

where at least one inequality is strict. We say that $s^{i}$ strictly dominates $t^{i}$, and $t^{i}$ is strictly dominated by $s^{i}$ if all of the above inequalities are strict. If $u^{i}\left(s^{i}, \mathbf{s}^{-i}\right)=u\left(t^{i}, \mathbf{s}^{-i}\right)$ for all $\mathbf{s}^{-i} \in \mathbf{S}^{-i}$, we will say that $s^{i}$ and $t^{i}$ are equivalent strategies for player $i$.

Any $\mu \in \Delta(\mathbf{S})$ is called a correlated strategy. Every mixed strategy profile $\mathbf{p} \in \mathbf{P}$ can be interpreted as the correlated strategy $\mu_{\mathbf{p}}$, where for every strategy profile $\mathbf{s} \in \mathbf{S}$,

10. For completeness, one can define the mediation value with costs as the ratio between the minimal cost at a mixed-strategy equilibrium to the minimal cost at a correlated equilibrium.

11. Interestingly, it can be shown that there exist classes of cost games in which the price of anarchy is bounded, while the price of anarchy with utilities in the analogous classes of utility games is unbounded. The class of cost games analogous to the class of utility games given in Example 2 in Section 4.1.2, constitutes one such example. 
$\mu_{\mathbf{p}}(\mathbf{s}) \triangleq \prod_{i=1}^{n} p^{i}\left(s^{i}\right)$. Whenever necessary we identify $\mathbf{p}$ with $\mu_{\mathbf{p}}$. With a slight abuse of notation, for every $\mu \in \Delta(\mathbf{S})$, we denote by $u^{i}(\mu)$ the expected utility of player $i$ when the correlated strategy $\mu \in \Delta(\mathbf{S})$ is played, that is:

$$
u^{i}(\mu)=\sum_{\mathbf{s} \in \mathbf{S}} u^{i}(\mathbf{s}) \mu(\mathbf{s})
$$

Naturally, for every $\mathbf{p} \in \mathbf{P}$ we denote $u^{i}(\mathbf{p})=u^{i}\left(\mu_{\mathbf{p}}\right)$. Hence $u^{i}(\mathbf{p})$ is the expected utility of player $i$ when the mixed strategy profile $\mathbf{p}$ is played.

We say that $\mathbf{p} \in \mathbf{P}$ is a mixed-strategy equilibrium if $u^{i}\left(\mathbf{p}^{-i}, p^{i}\right) \geq u^{i}\left(\mathbf{p}^{-i}, q^{i}\right)$ for every player $i \in N$ and for every $q^{i} \in P^{i}$. Let $\mathbf{p} \in \mathbf{P}$ be a mixed-strategy equilibrium. If for every $i, p^{i}$ is a pure strategy, we will also call $\mathbf{p}$ a pure-strategy equilibrium.

Definition 1 (Aumann, 1974, 1987) A correlated strategy $\mu \in \Delta(\mathbf{S})$ is a correlated equilibrium of $\Gamma$ if and only if for all $i \in N$ and all $s^{i}, t^{i} \in S^{i}$ :

$$
\sum_{\mathbf{s}^{-i} \in \mathbf{S}^{-i}} \mu\left(\mathbf{s}^{-i}, s^{i}\right)\left[u^{i}\left(\mathbf{s}^{-i}, s^{i}\right)-u^{i}\left(\mathbf{s}^{-i}, t^{i}\right)\right] \geq 0 .
$$

Consider a third party that picks randomly a pure-strategy profile $\mathbf{s} \in \mathbf{S}$ with respect to a well-known correlated strategy $\mu$, and recommends privately every player $i$ to play $s^{i}$. The left hand side of (2) captures the difference in expected utility between playing $s^{i}$, i.e., following the recommendation of the third party, and playing some other pure-strategy $t^{i}$ given that all other players follow their own recommendations. Hence, if this difference is nonnegative, player $i$ is better off playing $s^{i}$.

It is well-known and easily verified that every mixed-strategy equilibrium is a correlated equilibrium. For every correlated strategy $\mu$, let $u(\mu) \triangleq \sum_{i=1}^{n} u^{i}(\mu)$. The value $u(\mu)$ is called the welfare at $\mu$. Let $N(\Gamma)$ be the set of all mixed-strategy equilibria in $\Gamma$ and let $C(\Gamma)$ be the set of all correlated equilibria in $\Gamma$. We define $v_{C}(\Gamma)$ and $v_{N}(\Gamma)$ as follows:

$$
\begin{aligned}
& v_{C}(\Gamma) \triangleq \max \{u(\mu): \mu \in C(\Gamma)\}, \\
& v_{N}(\Gamma) \triangleq \max \{u(\mathbf{p}): \mathbf{p} \in N(\Gamma)\} .
\end{aligned}
$$

Note that $v_{N}(\Gamma)$ and $v_{C}(\Gamma)$ are well defined due to the compactness of $N(\Gamma)$ and $C(\Gamma)$ respectively, and the continuity of $u$. Define $\operatorname{opt}(\Gamma)$ (the maximal welfare) as follows:

$$
\operatorname{opt}(\Gamma) \triangleq \max \{u(\mu): \mu \in \Delta(\mathbf{S})\}=\max \{u(\mathbf{s}): \mathbf{s} \in \mathbf{S}\} .
$$

The mediation value of a nonnegative game $\Gamma$ is defined as follows:

$$
M V(\Gamma) \triangleq \frac{v_{C}(\Gamma)}{v_{N}(\Gamma)}
$$

If both $v_{N}(\Gamma)=0$ and $v_{C}(\Gamma)=0$, we define $M V(\Gamma)$ to be 1 . If $v_{N}(\Gamma)=0$ and $v_{C}(\Gamma)>0$, $M V(\Gamma)$ is defined to be $\infty$. Denote by $E V(\Gamma)$ the enforcement value of a nonnegative game $\Gamma$. That is,

$$
E V(\Gamma) \triangleq \frac{o p t(\Gamma)}{v_{C}(\Gamma)}
$$


If both $v_{C}(\Gamma)=0$ and $\operatorname{opt}(\Gamma)=0$, we define $E V(\Gamma)$ to be 1 . If $v_{C}(\Gamma)=0$ and $\operatorname{opt}(\Gamma)>0$, $E V(\Gamma)$ is defined to be $\infty$. Finally, for a class of nonnegative games $\mathcal{A}$, we define the mediation value and the enforcement value of this class as follows:

$$
M V(\mathcal{A}) \triangleq \sup _{\Gamma \in \mathcal{A}} M V(\Gamma) ; \quad \text { and } \quad E V(\mathcal{A}) \triangleq \sup _{\Gamma \in \mathcal{A}} E V(\Gamma)
$$

One of the tools we need in this paper is linear programming. For any game $\Gamma$ in strategic form, $C(\Gamma)$ is exactly the set of feasible solutions for the following linear program $\widehat{P}$. Moreover, $\mu \in C(\Gamma)$ is an optimal solution for $\widehat{P}$ if and only if $u(\mu)=v_{C}(\Gamma)$.

$$
\widehat{P}: \begin{cases}\max _{\mathbf{s} \in \mathbf{S}} \mu(\mathbf{s}) u(\mathbf{s}) & \\ \text { s.t. } & \forall \mathbf{s} \in \mathbf{S}, \\ \mu(\mathbf{s}) \geq 0 & \\ \sum_{\mathbf{s} \in \mathbf{S}} \mu(\mathbf{s})=1, & \forall i \in N, \forall\left(s^{i}, t^{i}\right) \in S^{i} \times S^{i}, \quad s^{i} \neq t^{i} . \\ \sum_{\mathbf{s}^{-i} \in \mathbf{S}^{-i}} \mu(\mathbf{s})\left[u^{i}\left(t^{i}, \mathbf{s}^{-i}\right)-u^{i}\left(\mathbf{s}^{\mathbf{i}}, \mathbf{s}^{-\mathbf{i}}\right)\right] \leq 0\end{cases}
$$

The dual problem of $\widehat{P}$ has one decision variable for each constraint in the primal. Let $\beta$ denote the dual variable associated with the primal constraint $\sum_{\mathbf{s} \in \mathbf{S}} \mu(\mathbf{s})=1$. Let $\alpha^{i}\left(t^{i} \mid s^{i}\right)$ denote the dual variable associated with the primal constraint defined by $\left(s^{i}, t^{i}\right)$, that is, by the constraint

$$
\sum_{\mathbf{s}^{-i} \in \mathbf{S}^{-i}} \mu(\mathbf{s})\left[u^{i}\left(t^{i}, \mathbf{s}^{-i}\right)-u^{i}\left(\mathbf{s}^{\mathbf{i}}, \mathbf{s}^{-\mathbf{i}}\right)\right] \leq 0
$$

and let $\alpha=\left(\alpha^{i}\right)_{i \in N}$, where $\alpha^{i}=\left(\alpha^{i}\left(t^{i} \mid s^{i}\right)\right)_{\left(s^{i}, t^{i}\right) \in S^{i} \times S^{i}, s^{i} \neq t^{i}}$. The dual problem can be written as follows:

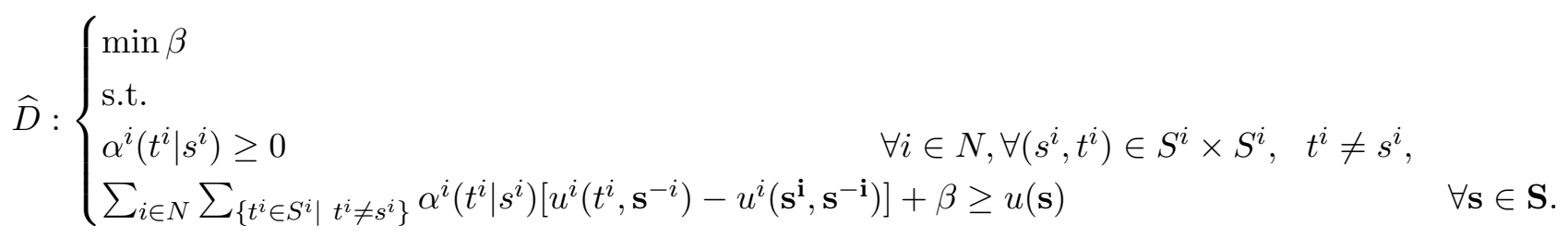

It is well known that problems $\widehat{P}$ and $\widehat{D}$ are feasible and bounded, and their objective values equal $v_{C}(\Gamma)$. The feasibility is a consequence of the existence of a mixed-strategy equilibrium proved by Nash (1951), and from the fact that every mixed-strategy equilibrium is also a correlated equilibrium. ${ }^{12}$

We will also make use of the following notation and definitions. Let $\mathcal{G}$ be the class of all nonnegative games in strategic form. For $m_{1}, m_{2}, \ldots, m_{n} \geq 1$ denote by $\mathcal{G}_{m_{1} \times m_{2} \times \cdots \times m_{n}} \subseteq \mathcal{G}$ the class of all games with $n$ players in which $\left|S^{i}\right|=m_{i}$ for every player $i$.

\section{Results for General Games}

The following two basic lemmas are used in some of the proofs in this paper. The proof of Lemma 1 follows directly from Definition 1, and the proof of Lemma 2 is standard. Therefore, these proofs are omitted.

12. An elementary proof of existence of correlated equilibrium, which does not use the existence of a mixedstrategy equilibrium is given by Hart and Schmeidler (1989). 
Lemma 1 Let $\Gamma$ be a game in strategic form. Let $s^{i} \in S^{i}$ be weakly dominated by some $t^{i} \in S^{i}$, and let $\mathbf{s}^{-i} \in \mathbf{S}^{-i}$. Then $\mu(\mathbf{s})=0$ for every correlated equilibrium $\mu$ for which

$$
u^{i}\left(t^{i}, \mathbf{s}^{-i}\right)>u^{i}\left(s^{i}, \mathbf{s}^{-i}\right) .
$$

Consequently, if $s^{i}$ is a strictly dominated strategy, $\mu(\mathbf{s})=0$ for every correlated equilibrium $\mu$.

Next we define extensions of a game by adding a dummy strategy to one of the players, or by adding a dummy player. Let $\Gamma \in \mathcal{G}_{m_{1} \times m_{2} \times \cdots \times m_{n}}$. The game $\tilde{\Gamma} \in \mathcal{G}_{m_{1} \times \cdots \times m_{i-1} \times\left(m_{i}+1\right) \times m_{i+1} \cdots \times m_{n}}$ is an extension of $\Gamma$ by adding a dummy strategy to player $i$ if it is obtained from $\Gamma$ by adding a strategy to player $i$ such that the utility of all $n$ players equal zero, when player $i$ uses this new strategy.

The game $\tilde{\Gamma} \in \mathcal{G}_{m_{1} \times m_{2} \cdots \times m_{n} \times 1}$ is an extension of $\Gamma$ by adding a dummy player, player $n+1$, if it is obtained from $\Gamma$ by adding player $n+1$ with a single strategy such that the utilities of this player are all zeros, and the utility of all $n$ players remain as in $\Gamma$. That is, $u^{i}(\mathbf{s}, d)=u^{i}(\mathbf{s})$ for every $\mathbf{s} \in S$, where $d$ denotes the unique added strategy of player $n+1$. A game $\tilde{\Gamma}$ is a trivial extension of $\Gamma$ if there is a sequence of games,

$$
\Gamma=\Gamma_{0}, \Gamma_{1}, \cdots, \Gamma_{t}=\tilde{\Gamma}
$$

such that $\Gamma_{k}$ is obtained from $\Gamma_{k-1}$ by adding a dummy player or a dummy strategy.

Lemma 2 Let $\tilde{\Gamma}$ be a trivial extension of $\Gamma$. Then, $M V(\tilde{\Gamma})=M V(\Gamma)$ and $E V(\tilde{\Gamma})=$ $E V(\Gamma)$.

\subsection{The Mediation Value}

In this section we show the power of correlation in general games. We start with extending Aumann's result on the power of correlation in $2 \times 2$ games.

\subsubsection{TWO-PERSON TWO-STRATEGY GAMES}

Aumann's example shows that a mediation value of $\frac{10}{9}$ can be obtained in a $2 \times 2$ game. We show:

Theorem $1 M V\left(\mathcal{G}_{2 \times 2}\right)=\frac{4}{3}$.

The following lemma is needed for the proof of Theorem 1:

Lemma 3 (Peeters 8 Potters, 1999) Let $\Gamma \in \mathcal{G}_{2 \times 2}$. If there exist a correlated equilibrium in $\Gamma$, which is not induced by a mixed-strategy equilibrium, $\Gamma$ has at least two pure-strategy equilibria.

Before we give the proof of Theorem 1 we need the following technical remarks, which holds for the rest of the paper:

Remark: In all the games introduced by figures, we denote by player 1,2,3 the row, column, and matrix (if exist) players respectively. In each strategy profile the players' utilities are given from left to right where player $i$ 's utility is the $i^{\text {th }}$ left payoff.

Proof of Theorem 1: We begin by showing that $M V\left(\mathcal{G}_{2 \times 2}\right) \leq \frac{4}{3}$. Figure 1 below describes an arbitrary game $\Gamma \in \mathcal{G}_{2 \times 2}$. 


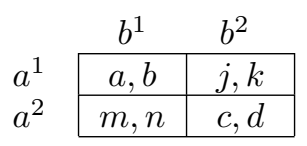

Figure 1

By Lemma 3, If $\Gamma$ has less than two pure-strategy equilibrium profiles, $M V(\Gamma)=1$. Therefore, we can assume without loss of generality that $\Gamma$ has two or more pure-strategy equilibrium profiles. However, if there are four pure-strategy equilibrium profiles, the mediation value equals 1 . Therefore we should discuss the cases in which $\Gamma$ has either two or three pure-strategy equilibrium profiles.

Suppose $\Gamma$ possesses exactly three pure-strategy equilibrium profiles, and without loss of generality let $\left(a^{2}, b^{2}\right)$ be the only strategy profile not in equilibrium. Since $\left(a^{1}, b^{1}\right),\left(a^{2}, b^{1}\right)$ and $\left(a^{1}, b^{2}\right)$ are all pure-strategy equilibria, $m=a$ and $b=k$. Because $\left(a^{2}, b^{2}\right)$ is not an equilibrium, $c<j$ or $d<n$. If $c<j$, by Lemma 1 , every correlated equilibrium $\mu \in C(\Gamma)$ satisfies $\mu\left(a^{2}, b^{2}\right)=0$, and therefore $M V(\Gamma)=1$. Similarly, if $d<n, \mu\left(a^{2}, b^{2}\right)=0$ implies $M V(\Gamma)=1$.

Suppose there are exactly two pure-strategy equilibrium profiles in $\Gamma$. These two equilibrium profiles may be in the same row, in the same column, or on a diagonal. Obviously, the proof for the case that the two pure-strategy equilibrium profiles are in the same row or in the same column is covered by the following proof, which assumes that the two purestrategy equilibrium profiles are in the first row. That is, $\left(a^{1}, b^{1}\right)$ and $\left(a^{1}, b^{2}\right)$ are both pure-strategy equilibria. This assumption implies that $b=k$. Observe that any strategy profile in which player 1 plays strategy $a^{1}$ with probability one, and player 2 plays any mixed strategy $p^{2} \in P^{2}$, is a mixed-strategy equilibrium. Since there are exactly two purestrategy equilibria, it must be that $m<a$ or $c<j$. If $m<a$ and $c<j$, by Lemma 1 every correlated equilibrium $\mu \in C(\Gamma)$ satisfies both $\mu\left(a^{2}, b^{1}\right)=0$ and $\mu\left(a^{2}, b^{2}\right)=0$. Therefore $M V(\Gamma)=1$. Suppose $m=a$. Therefore $c<j$. Hence, by Lemma 1 every correlated equilibrium $\mu \in C(\Gamma)$ satisfies $\mu\left(a^{2}, b^{2}\right)=0$. Since $\left(a^{2}, b^{1}\right)$ is not a pure-strategy equilibrium, $n<d$. Since $b=k$ and $n<d$, then again by Lemma 1 , every correlated equilibrium $\mu \in C(\Gamma)$ satisfies $\mu\left(a^{2}, b^{1}\right)=0$. Therefore $M V(\Gamma)=1$. We showed that $M V(\Gamma)=1$ if there are two pure-strategy equilibrium profiles, and they are on the same row or the same column.

We proceed to the last case in which the two pure-strategy equilibrium profiles are on a diagonal. Without loss of generality let $\left(a^{1}, b^{1}\right)$ and $\left(a^{2}, b^{2}\right)$ be pure-strategy equilibrium profiles. It was shown by Peeters and Potters (1999) that if $c=j$ or $a=m$ or $b=k$ or $d=n, C(\Gamma)$ is exactly the convex hull of $N(\Gamma)$. Hence, in this case there is no extreme point of $C(\Gamma)$ that is not a mixed-strategy equilibrium, and therefore the mediation value equals 1 . Therefore we can assume:

$$
c>j, a>m, b>k \text { and } d>n .
$$

If both, $u\left(a^{1}, b^{2}\right)$ and $u\left(a^{2}, b^{1}\right)$ are smaller than $\max \left\{u\left(a^{1}, b^{1}\right), u\left(a^{2}, b^{2}\right)\right\}, M V(\Gamma)=1$ and the proof is completed. Therefore, without loss of generality we make the following two assumptions:

(A1) $u\left(a^{1}, b^{1}\right) \leq u\left(a^{2}, b^{2}\right)$. That is, $a+b \leq c+d$. 


$$
u\left(a^{2}, b^{1}\right) \geq u\left(a^{2}, b^{2}\right) . \text { That is, } m+n \geq c+d .
$$

Hence, the set of mixed-strategy equilibria is:

$$
N(\Gamma)=\left\{((1,0),(1,0)),((0,1),(0,1)),\left(\left(\frac{1}{1+\beta}, \frac{\beta}{1+\beta}\right),\left(\frac{1}{1+\alpha}, \frac{\alpha}{1+\alpha}\right)\right)\right\},
$$

where $\alpha=\frac{a-m}{c-j}$ and $\beta=\frac{b-k}{d-n}$. Note that because of (3), $\alpha$ and $\beta$ are positive.

Before continuing with the proof we will need the following geometric characterization of $C(\Gamma)$. By Peeters and Potters (1999), $C(\Gamma)$ is a polyhedron with the following five extreme points $\mu_{i}, \quad i=1, \ldots, 5$ :

$$
\begin{aligned}
& \mu_{1}=\left(\begin{array}{ll}
1, & 0 \\
0, & 0
\end{array}\right), \mu_{2}=\left(\begin{array}{ll}
0, & 0 \\
0, & 1
\end{array}\right), \mu_{3}=\left(\begin{array}{cc}
\frac{1}{(1+\alpha)(1+\beta)}, & \frac{\alpha}{(1+\alpha)(1+\beta)} \\
\frac{\beta}{(1+\alpha)(1+\beta)}, & \frac{\alpha \beta}{(1+\alpha)(1+\beta)}
\end{array}\right), \\
& \mu_{4}=\left(\begin{array}{cc}
\frac{1}{(1+\alpha+\alpha \beta)}, & \frac{\alpha}{(1+\alpha+\alpha \beta)} \\
0, & \frac{\alpha \beta}{(1+\alpha+\alpha \beta}
\end{array}\right), \mu_{5}=\left(\begin{array}{cc}
\frac{1}{(1+\beta+\alpha \beta)}, & 0 \\
\frac{\beta}{(1+\beta+\alpha \beta)}, & \frac{\alpha \beta}{(1+\beta+\alpha \beta)}
\end{array}\right),
\end{aligned}
$$

where $\mu_{i}(j, k)$ denotes the probability given to the strategy profile $\left(a^{j}, b^{k}\right)$ in the correlated equilibrium $\mu_{i}$. That is, the $(j, k)^{t h}$ entry of $\mu_{i}$ is $\mu_{i}\left(a^{j}, b^{k}\right)$. By our agreement to identify mixed-strategy profiles with correlated strategies we observe that the set of mixed-strategy equilibrium is precisely

$$
N(\Gamma)=\left\{\mu_{1}, \mu_{2}, \mu_{3}\right\} .
$$

We have to prove that $u(\mu) \leq \frac{4}{3} v_{N}(\Gamma)$ for every correlated equilibrium $\mu \in C(\Gamma)$. It is sufficient to prove that the inequality holds for the extreme points of $C(\Gamma)$. Since $\mu_{1}, \mu_{2}$ and $\mu_{3}$ are mixed-strategy equilibria, $u\left(\mu_{i}\right) \leq v_{N}(\Gamma)$ for $i=1,2,3$. Therefore it suffices to prove the inequality for $\mu_{4}$ and $\mu_{5}$.

We first derive a couple of inequalities which will be useful for us. By $(A 1)$ and since all utilities are nonnegative, $a \leq c+d$ and $d \leq c+d$. Therefore, since $m<a$ and $n<d$, we obtain the inequality

$$
m+n \leq 2(c+d)
$$

Since $\left(a^{1}, b^{1}\right)$ and $\left(a^{2}, b^{2}\right)$ are the only pure-strategy equilibrium profiles, $m+n \leq a+d$ and $j+k \leq c+b$. By $(A 2)$ and since $m+n \leq a+d, c \leq a$. Hence $j+k \leq a+b$. Therefore, by $(A 1)$ we obtain

$$
j+k \leq c+d .
$$

Note that inequality (6) implies that $u\left(\mu_{4}\right) \leq v_{N}(\Gamma)$ since $\left(a^{2}, b^{2}\right)$ is a pure-strategy equilibrium.

It remains to show that $u\left(\mu_{5}\right) \leq v_{N}(\Gamma)$. By $(A 1), u\left(\mu_{1}\right) \leq u\left(\mu_{2}\right)$. We distinguish between the following two cases:

Case 1: $u\left(\mu_{3}\right) \geq u\left(\mu_{2}\right)$.

By $(A 1), u\left(\mu_{1}\right) \leq u\left(\mu_{2}\right)$. Therefore, by $(4), v_{N}(\Gamma)=u\left(\mu_{3}\right)$.

Hence,

$$
\frac{u\left(\mu_{5}\right)}{v_{N}(\Gamma)}=\frac{a+b+(m+n) \beta+(c+d) \alpha \beta}{1+\beta+\alpha \beta} \times \frac{1+\beta+\alpha+\alpha \beta}{a+b+(j+k) \alpha+(m+n) \beta+(c+d) \alpha \beta} .
$$


Therefore, because $(j+k) \alpha \geq 0$,

$$
\frac{u\left(\mu_{5}\right)}{v_{N}(\Gamma)} \leq \frac{1+\beta+\alpha+\alpha \beta}{1+\beta+\alpha \beta}
$$

For $z>0$, let $f_{1}(z)=\frac{1+\beta+z+z \beta}{1+\beta+z \beta}$. Hence, it suffices to show that $f_{1}(\alpha) \leq \frac{4}{3}$.

Let $K=\frac{m+n-c-d}{c+d-j-k}$. Since $\left(a^{1}, b^{1}\right)$ and $\left(a^{2}, b^{2}\right)$ are both pure-strategy equilibrium profiles,

$$
m+n-c-d \leq a-c, \quad c+d-j-k \geq d-b .
$$

Therefore by $(A 1)$,

$$
K \leq 1
$$

Since $u\left(\mu_{3}\right) \geq c+d$,

$$
a+b+(j+k) \alpha+(m+n) \beta \geq(c+d)(1+\alpha+\beta) .
$$

Therefore

$$
\alpha \leq \frac{\beta(m+n-c-d)+a+b-c-d}{c+d-j-k} .
$$

By Equation (7) and $(A 1), \alpha \leq \beta K$. Since, $K \leq 1, \alpha \leq \beta$. Note that $f_{1}(z)$ is non-decreasing in $z>0$. Therefore,

$$
f_{1}(\alpha) \leq f_{1}(\beta)=\frac{1+2 \beta+\beta^{2}}{1+\beta+\beta^{2}}
$$

Since $\frac{1+2 \beta+\beta^{2}}{1+\beta+\beta^{2}}=\frac{(\beta+1)^{2}}{(\beta+1)^{2}-\beta}$ is maximized for $\beta>0$ at $\beta=1$,

$$
f_{1}(\alpha) \leq \frac{4}{3}
$$

Case 2: $u\left(\mu_{3}\right)<u\left(\mu_{2}\right)$.

By $(A 1), u\left(\mu_{1}\right) \leq u\left(\mu_{2}\right)$. Therefore, by $(4), v_{N}(\Gamma)=u\left(\mu_{2}\right)$. Therefore,

$$
\frac{u\left(\mu_{5}\right)}{v_{N}(\Gamma)}=\frac{a+b+(m+n) \beta+(c+d) \alpha \beta}{(1+\beta+\alpha \beta)(c+d)} .
$$

For $z>0$ let $f_{2}(z)=\frac{a+b+(m+n) \beta+(c+d) z \beta}{(1+\beta+z \beta)(c+d)}$. Hence, It suffices to show that $f_{2}(\alpha) \leq \frac{4}{3}$. Not that in this case the inequality (7) is reversed. That is

$$
\alpha \geq \frac{\beta(m+n-c-d)+a+b-c-d}{c+d-j-k},
$$

and since $j, k \geq 0$

$$
\alpha \geq \frac{\beta(m+n-c-d)+a+b-c-d}{c+d} .
$$

We distinguish between the following two cases, noticing that $f_{2}(x)$ is non-increasing for $x>0$ : 
Case 2.1: $a+b=c+d$ : In this case, because $f_{2}$ is non-increasing and (10) holds,

$$
f_{2}(\alpha) \leq \frac{c+d+(m+n) \beta+(m+n-c-d) \beta^{2}}{\left(1+\beta+\frac{(m+n-c-d)}{c+d} \beta^{2}\right)(c+d)} .
$$

Set $x=c+d$. Therefore, by $(A 2)$, there exists $1 \leq t \leq 2$ for which $t x=m+n$. By (11)

$$
f_{2}(\alpha) \leq \frac{x+t x \beta+(t-1) x \beta^{2}}{x+x \beta+(t-1) x \beta^{2}}=\frac{1+t \beta+(t-1) \beta^{2}}{1+\beta+(t-1) \beta^{2}} \leq \frac{4}{3}
$$

where the last inequality follows from similar arguments to those following (8).

Case 2.2: $a+b<c+d$ :

Set $x=a+b$. Therefore by (A1), (A2) and (5), for some $t>1$ and for some $1 \leq k \leq 2$,

$$
c+d=t x \quad \text { and } \quad m+n=k t x .
$$

Hence,

$$
k t=\frac{m+n}{a+b} \leq \frac{a+b+c+d}{a+b}=t+1,
$$

where the inequality follows since $m+n \leq a+b+c+d$. Therefore

$$
t \leq \frac{1}{k-1}
$$

Therefore

$$
\begin{gathered}
f_{2}(\alpha) \leq \frac{a+b+(m+n) \beta+(c+d)\left[\frac{\beta(m+n-c-d)+a+b-c-d}{c+d}\right] \beta}{\left(1+\beta+\frac{\beta(m+n-c-d)+a+b-c-d}{c+d} \beta\right)(c+d)}= \\
\frac{1+k t \beta+t(k-1) \beta^{2}+\beta-t \beta}{t+t \beta+t(k-1) \beta^{2}+\beta-t \beta}=\frac{1+\beta+t \beta(\beta+1)(k-1)}{t+t \beta^{2}(k-1)+\beta} \leq \\
\frac{1+\beta^{2}+2 \beta}{1+\beta^{2}(k-1)+\beta} \leq \frac{(\beta+1)^{2}}{(\beta+1)^{2}-\beta} \leq \frac{4}{3},
\end{gathered}
$$

where the right inequality in (15) follows from (14), the left inequality in (16) follows since $1 \leq k \leq 2$, and the right inequality in (16) follows since the maximum value of $\frac{(\beta+1)^{2}}{(\beta+1)^{2}-\beta}$ is attained at $\beta=1$.

We showed that the mediation value is bounded from above by $\frac{4}{3}$. It remains to show that this bound is tight. We show a family of games for which the mediation value approaches the above $\frac{4}{3}$ bound. Consider the family of games $\Gamma_{x}$ shown in Figure 2 (a variant of Aumann's example), where $x>1$. 


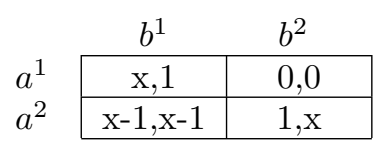

Figure 2

In this game the strategy profiles $\left(a^{1}, b^{1}\right)$ and $\left(a^{2}, b^{2}\right)$ are both pure-strategy equilibrium profiles, and $u\left(a^{1}, b^{1}\right)=u\left(a^{2}, b^{2}\right)=x+1$. There exists one more mixed-strategy equilibrium, in which each player assigns the probability 0.5 to each of her strategies, which yields a welfare lower than $x+1$. The correlated strategy $\mu \in \Delta(\mathbf{S})$, where each of the strategy profiles $\left(a^{1}, b^{1}\right),\left(a^{2}, b^{1}\right)$ and $\left(a^{2}, b^{2}\right)$ is played with equal probability $1 / 3$ is a correlated equilibrium and $u(\mu)=\frac{4 x}{3}$. Hence $M V\left(\Gamma_{x}\right) \geq \frac{4 x}{3(x+1)}$. Therefore $M V\left(\Gamma_{x}\right) \rightarrow \frac{4}{3}$ when $x \rightarrow \infty$.

\subsubsection{General Games}

Theorem 1 shows that the mediation value of the class of nonnegative $2 \times 2$ games is finite. In the next theorem we show that the mediation value is unbounded as we move slightly beyond $2 \times 2$ games. In particular, if one of the players in a 2-player game has at least three strategies, while the other remains with two strategies, the mediation value is already unbounded. Similarly, if there are three players each with two strategies, the mediation value is again unbounded. ${ }^{13}$

Theorem $2 M V\left(\mathcal{G}_{m_{1} \times m_{2}}\right)=\infty$ for every $m_{1}, m_{2} \geq 2$ for which $\max \left(m_{1}, m_{2}\right) \geq 3$.

Proof: By Lemma 2 it suffices to prove that $M V\left(\mathcal{G}_{3 \times 2}\right)=\infty$. Let $\Gamma_{x, \epsilon}$ be the following parametric $\mathcal{G}_{2 \times 3}$ game In Figure 3,

\begin{tabular}{|c|c|c|c|}
\hline & $b^{1}$ & $b^{2}$ & $b^{3}$ \\
\hline$a^{1}$ & $x, 1-\epsilon$ & $z, 1$ & 0,0 \\
\hline$a^{2}$ & $0, z-\epsilon$ & $z-1, z-1$ & $1, z$ \\
\hline
\end{tabular}

Figure 3

where $z>2$ is fixed, $x>z$ and $0<\epsilon<0.5$.

We first show that

$$
N\left(\Gamma_{x, \epsilon}\right)=\left\{((1,0)(0,1,0)),((0,1),(0,0,1)),\left((\epsilon, 1-\epsilon),\left(\frac{1}{1+x}, 0, \frac{x}{1+x}\right)\right)\right\} .
$$

It is standard to check that each profile in $N\left(\Gamma_{x, \epsilon}\right)$ is indeed a mixed-strategy equilibrium, and that if player 1 plays a pure-strategy in equilibrium, the only mixed-strategy equilibrium are $((1,0)(0,1,0))$ and $((0,1),(0,0,1))$. We have to show that if player 1 plays fully mixed (that is, he assigns positive probabilities to both $a^{1}$ and $\left.a^{2}.\right),\left((\epsilon, 1-\epsilon),\left(\frac{1}{1+x}, 0, \frac{x}{1+x}\right)\right)$ is the only mixed-strategy equilibrium. Indeed, note that if player 2 plays a pure strategy, player 1 is never indifferent between $a^{1}$ and $a^{2}$ and therefore player 1 is always better off deviating to some pure strategy. If player 2 assigns positive probability only to $b^{1}$ and $b^{2}$, player 1 is better off playing $a^{1}$. If player 2 assigns positive probability only to $b^{2}$ and $b^{3}$, the only

13. The results presented in this paper showing that the mediation value may be $\infty$, can also be established when we assume that the utilities are uniformly bounded, e.g. when all utilities are in the interval $[0,1]$. 
mixed-strategy player 1 can play in order that player 2 will be indifferent between $b^{2}$ and $b^{3}$ is $(1 / 2,1 / 2)$, but then player 2 is better off playing $b^{1}$. Similarly, it can be shown that there does not exist an equilibrium in which player 1 fully mixes and player 2 assigns positive probabilities to all of her pure strategies. Suppose player 2 assigns positive probabilities only to $b^{1}$ and $b^{3}$. If the probability that player 2 assigns to $b^{1}$ is higher (lower) than $\frac{1}{1+x}$, player 1 is better off playing $a^{1}\left(a^{2}\right)$. Hence, player 2 plays $\left(\frac{1}{1+x}, 0, \frac{x}{1+x}\right)$ in equilibrium in which player 1 fully mixes, and therefore It is standard to prove that player 1 must play $(\epsilon, 1-\epsilon)$.

Next we show that $v_{N}\left(\Gamma_{x, \epsilon}\right) \leq z+2$ for small enough $\epsilon$. Notice that the welfare in both pure-strategy equilibria is $z+1$. The welfare in the mixed-strategy equilibrium $((\epsilon, 1-$ $\epsilon),\left(\frac{1}{1+x}, 0, \frac{x}{1+x}\right)$ is:

$$
\begin{gathered}
\frac{\epsilon(x+1-\epsilon)+(1-\epsilon)(z-\epsilon)+x(1-\epsilon)(z+1)}{x+1}= \\
z-z \epsilon+\frac{x}{x+1} .
\end{gathered}
$$

Note that $z-z \epsilon+\frac{x}{x+1} \rightarrow z+1$ as $x \rightarrow \infty$ and $\epsilon \rightarrow 0$.

We proceed to show a net of games, $\Gamma_{x, \epsilon}$ such that $v_{C}\left(\Gamma_{x, \epsilon}\right) \rightarrow \infty$ as $\epsilon \rightarrow 0$ and $x \rightarrow \infty$. Let $\mu$ be a correlated strategy. $\mu$ is a correlated equilibrium in $\Gamma_{x, \epsilon}$ if and only if the following 9 inequalities are satisfied (in brackets we relate the inequality here to the corresponding inequality in (2)):

1. $\mu\left(a^{1}, b^{1}\right) x+\mu\left(a^{1}, b^{2}\right)-\mu\left(a^{1}, b^{3}\right) \geq 0 . \quad\left(i=1, s^{i}=a^{1}, t^{i}=a^{2}\right)$

2. $\mu\left(a^{2}, b^{3}\right)-\mu\left(a^{2}, b^{2}\right)-x \mu\left(a^{2}, b^{1}\right) \geq 0 . \quad\left(i=1, s^{i}=a^{2}, t^{i}=a^{1}\right)$

3. $\mu\left(a^{1}, b^{1}\right) \leq \frac{(1-\epsilon) \mu\left(a^{2}, b^{1}\right)}{\epsilon} . \quad\left(i=2, s^{i}=b^{1}, t^{i}=b^{2}\right)$

4. $\mu\left(a^{1}, b^{1}\right) \geq \frac{\epsilon \mu\left(a^{2}, b^{1}\right)}{1-\epsilon} . \quad\left(i=2, s^{i}=b^{1}, t^{i}=b^{3}\right)$

5. $\mu\left(a^{1}, b^{2}\right) \geq \frac{(1-\epsilon) \mu\left(a^{2}, b^{2}\right)}{\epsilon} . \quad\left(i=2, s^{i}=b^{2}, t^{i}=b^{1}\right)$

6. $\mu\left(a^{1}, b^{2}\right) \geq \mu\left(a^{2}, b^{2}\right) . \quad\left(i=2, s^{i}=b^{2}, t^{i}=b^{3}\right)$

7. $\mu\left(a^{2}, b^{3}\right) \geq \frac{(1-\epsilon) \mu\left(a^{1}, b^{3}\right)}{\epsilon} . \quad\left(i=2, s^{i}=b^{3}, t^{i}=b^{1}\right)$

8. $\mu\left(a^{2}, b^{3}\right) \geq \mu\left(a^{1}, b^{3}\right) . \quad\left(i=2, s^{i}=b^{3}, t^{i}=b^{2}\right)$

9. $\sum_{i=1}^{2} \sum_{j=1}^{3} \mu\left(a^{i}, b^{j}\right)=1$.

Set $\mu\left(a^{1}, b^{1}\right)=\epsilon, \mu\left(a^{2}, b^{1}\right)=2 \epsilon^{2}, \mu\left(a^{1}, b^{2}\right)=\epsilon(1-\epsilon), \mu\left(a^{1}, b^{3}\right)=\mu\left(a^{2}, b^{2}\right)=\epsilon^{2}, \mu\left(a^{2}, b^{3}\right)=$ $1-\mu\left(a^{1}, b^{3}\right)-\sum_{i=1}^{2} \sum_{j=1}^{2} \mu\left(a^{i}, b^{j}\right)$ and let $x=\frac{1}{4 \epsilon^{2}}$. Let $\epsilon \rightarrow 0$. All nine constraints are satisfied for small enough $\epsilon$. Note that $\lim _{\epsilon \rightarrow 0} x \mu\left(a^{1}, b^{1}\right)=\infty$. Since $M V\left(\Gamma_{x, \epsilon}\right) \geq x \mu\left(a^{1}, b^{1}\right)$ we obtain $M V\left(\Gamma_{x, \epsilon}\right) \rightarrow \infty$ as $\epsilon \rightarrow 0$.

Theorem $3 M V\left(\mathcal{G}_{m_{1} \times \cdots \times m_{n}}\right)=\infty$ for every $n \geq 3$ and for every $m_{1}, m_{2}, \ldots, m_{n} \geq 2$. 
Proof: By Lemma 2 it suffices to prove that $M V\left(\mathcal{G}_{2 \times 2 \times 2}\right)=\infty$. Consider the following three player game $\Gamma$ (Figure 4$)$ :

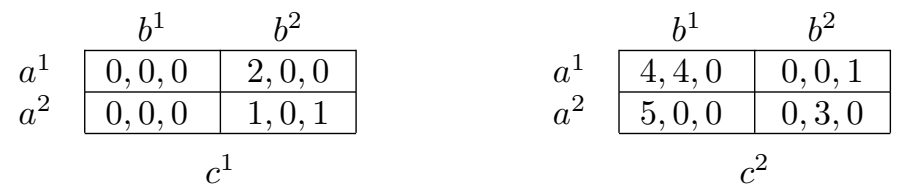

Figure 4

We will show that $v_{N}(\Gamma)=0$, that is, the welfare in every mixed-strategy equilibrium is zero. In addition we will construct a correlated equilibrium, which yields a strictly positive welfare. We begin with proving that $v_{N}(\Gamma)=0$. Note that the only pure-strategy equilibrium profiles in the game are $\left(a^{1}, b^{1}, c^{1}\right)$ and $\left(a^{2}, b^{1}, c^{1}\right)$. Obviously, every strategy profile in which players 2 and 3 play $b^{1}$ and $c^{1}$ respectively, and player 1 plays any mixed strategy, is a mixed-strategy equilibrium. We next show that there are no other mixedstrategy equilibria in the game. First we show that there are no other mixed-strategy equilibria in which at least one of the players plays a pure strategy. We verify this for each player:

1. Assume player 3 plays $c^{2}$ with probability one. If $1>p^{2}\left(b^{1}\right)>0, p^{1}\left(a^{2}\right)=1$, but then player 3 is better off playing $c^{1}$. If $p^{2}\left(b^{1}\right)=1, p^{1}\left(a^{2}\right)=1$, but then player 2 is better off playing $b^{2}$. If $p^{2}\left(b^{2}\right)=1$, player 1 is indifferent. If $p^{1}\left(a^{1}\right) \geq 0.5$, player 2 is better off playing $b^{1}$. If $p^{1}\left(a^{1}\right)<0.5$, player 3 is better off playing $c^{1}$.

2. Assume player 3 plays $c^{1}$ with probability one. If $p^{2}\left(b^{2}\right)>0, p^{1}\left(a^{1}\right)=1$, but then player 3 is better off playing $c^{2}$.

3. Assume player 2 plays $b^{1}$ with probability one. Player 3 is indifferent between her strategies. If $p^{3}\left(c^{2}\right)>0, p^{1}\left(a^{2}\right)=1$, but then player 2 is better off playing $b^{2} \cdot p^{3}\left(c^{1}\right)=$ 1 has been dealt in the previous case. Assume player 2 plays $b^{2}$ with probability one. If $p^{3}\left(c^{1}\right)>0, p^{1}\left(a^{1}\right)=1$, but then player 3 is better off playing $c^{2}$.

4. Assume player 1 plays $a^{1}$ with probability one. If $p^{2}\left(b^{2}\right)>0, p^{3}\left(c^{2}\right)=1$, but then player 2 is better off playing $b^{1}$ with probability one. Assume player 1 plays $a^{2}$ with probability one. If $p^{3}\left(c^{2}\right)>0, p^{2}\left(b^{2}\right)=1$, but then player 3 is better off playing $c^{1}$ with probability one.

Next we prove that there does not exist a completely mixed-strategy equilibrium, (an equilibrium in which every player assigns a positive probability to all of her strategies). Suppose in negation that this is not the case. Let $((p, 1-p),(q, 1-q),(h, 1-h))$ be a completely mixed-strategy equilibrium, that is $1>p, q, h>0$. By the equilibrium properties, player 2 is indifferent between $b^{1}$ and $b^{2}$ given that players 1 and 3 play $(p, 1-p)$ and $(h, 1-h)$ respectively. Hence $4 p(1-h)=3(1-p)(1-h)$, which implies that $p=\frac{3}{7}$. Similarly, player 3 is indifferent between $c^{1}$ and $c^{2}$ given that players 1 and 2 play $(p, 1-p)$ and $(q, 1-q)$ respectively. Therefore $(1-p)(1-q)=p(1-q)$ yielding $p=0.5$, which is a contradiction. Therefore, there does not exist a completely mixed-strategy equilibrium.

We proved that $v_{N}(\Gamma)=0$. It remains to prove that there exists a correlated equilibrium with a a strictly positive welfare; this will imply that the mediation value is infinity. Let 
$\mu \in \Delta(\mathbf{S})$ be the following correlated strategy. $\mu\left(a^{1}, b^{2}, c^{1}\right)=\mu\left(a^{2}, b^{2}, c^{1}\right)=\mu\left(a^{1}, b^{1}, c^{2}\right)=$ $\mu\left(a^{2}, b^{1}, c^{2}\right)=0.25$ and for all other $\mathbf{s} \in \mathbf{S}, \mu(\mathbf{s})=0$. We proceed to prove that $\mu$ is a correlated equilibrium. Indeed, observe that the following inequalities, which define a correlated equilibrium are satisfied (as usual, in brackets we relate the inequality here to the corresponding inequality in (2)):

1. $\mu\left(a^{1}, b^{2}, c^{1}\right)(2-1)+\mu\left(a^{1}, b^{1}, c^{2}\right)(4-5) \geq 0 . \quad\left(i=1, s^{i}=a^{1}, t^{i}=a^{2}\right)$

2. $\mu\left(a^{2}, b^{2}, c^{1}\right)(1-2)+\mu\left(a^{2}, b^{1}, c^{2}\right)(5-4) \geq 0 . \quad\left(i=1, s^{i}=a^{2}, t^{i}=a^{1}\right)$

3. $\mu\left(a^{1}, b^{2}, c^{1}\right)(0-0)+\mu\left(a^{2}, b^{2}, c^{1}\right)(0-0) \geq 0 . \quad\left(i=2, s^{i}=b^{2}, t^{i}=b^{1}\right)$

4. $\mu\left(a^{1}, b^{1}, c^{2}\right)(4-0)+\mu\left(a^{2}, b^{1}, c^{2}\right)(0-3) \geq 0 . \quad\left(i=2, s^{i}=b^{1}, t^{i}=b^{2}\right)$

5. $\mu\left(a^{1}, b^{2}, c^{1}\right)(0-1)+\mu\left(a^{2}, b^{1}, c^{1}\right)(1-0) \geq 0 . \quad\left(i=3, s^{i}=c^{1}, t^{i}=c^{2}\right)$

6. $\mu\left(a^{1}, b^{1}, c^{2}\right)(0-0)+\mu\left(a^{2}, b^{1}, c^{2}\right)(0-0) \geq 0 . \quad\left(i=3, s^{i}=c^{2}, t^{i}=c^{1}\right)$

The proof of Theorem 3 was based on showing that there exists a three-player nonnegative game, in which not all utilities are zero, yet the welfare in every mixed-strategy equilibrium is zero. The next lemma shows that this phenomenon can not happen in a two player game.

Lemma 4 Let $\Gamma \in \mathcal{G}_{n \times m}, n, m \geq 1 . v_{N}(\Gamma)=0$ implies that all the utilities in $\Gamma$ are zero. That is, for $i=1,2$,

$$
u^{i}\left(t^{1}, t^{2}\right)=0 \quad \forall t^{1} \in S^{1}, \forall t^{2} \in S^{2} .
$$

Proof: The proof is by induction on the total number of pure strategies $n+m$ in the game. First note that the assertion holds for all games, $\Gamma \in \mathcal{G}_{n \times m}$ for which $n+m=2$ because in this case each player has exactly one strategy. Let $k \geq 2$, and assume the assertion holds for every game, $\Gamma \in \mathcal{G}_{n \times m}$ for which $n+m \leq k$.

Let $\Gamma \in \mathcal{G}_{n \times m}$ be a game in which $n+m=k+1$ and for which $v_{N}(\Gamma)=0$. We should prove that $\Gamma$ is the zero game. As $k+1 \geq 3$, there exists at least one player that has more than one strategy; without loss of generality, player 1 is such a player.

Let $\mathbf{p}=\left(p^{1}, p^{2}\right)$ be a mixed-strategy equilibrium in $\Gamma$, that is, $\mathbf{p} \in N(\Gamma)$. Because $v_{N}(\Gamma)=0$, the welfare at $\mathbf{p}$ equals 0 . That is, $u(\mathbf{p})=u^{1}(\mathbf{p})+u^{2}(\mathbf{p})=0$. Because utilities are nonnegative,

$$
u^{1}(\mathbf{p})=0=u^{2}(\mathbf{p})
$$

Let $s^{1}$ be an arbitrary fixed strategy for player 1 for which $p^{1}\left(s^{1}\right)>0$, and let $s^{2}$ be an arbitrary fixed strategy for player 2 for which $p^{2}\left(s^{2}\right)>0$.

Claim 1: (i) $u^{1}\left(t^{1}, s^{2}\right)=0 \quad \forall t^{1} \in S^{1}$. (ii) $u^{2}\left(s^{1}, t^{2}\right)=0 \quad \forall t^{2} \in S^{2}$.

Proof of Claim 1. Let $t^{1} \in S^{1}$. Since $\left(p^{1}, p^{2}\right)$ is a mixed-strategy equilibrium, $u^{1}\left(t^{1}, p^{2}\right) \leq$ $u^{1}\left(p^{1}, p^{2}\right)=0$. Since $u^{1}$ is nonnegative, $u^{1}\left(t^{1}, p^{2}\right)=0$. Since $u^{1}\left(t^{1}, p^{2}\right)=\sum_{t^{2} \in S^{2}} p^{2}\left(t^{2}\right) u^{1}\left(t^{1}, t^{2}\right)$, $u^{1}$ is nonnegative, and $p^{2}\left(s^{2}\right)>0, u^{1}\left(t^{1}, s^{2}\right)=0$. This proves (i). (ii) is similarly proved.

Consider the game $\Gamma_{*} \in \mathcal{G}_{(n-1) \times m}$ obtained by removing $s^{1}$ from $S^{1}$; In this game the strategy set of player 1 is $T^{1}=S^{1} \backslash\left\{s^{1}\right\}$ and the strategy set of player 2 remains $S^{2}$. With a 
slight abuse of notations The utility functions in $\Gamma_{*}$ are also denoted by $u^{1}$ and $u^{2}$. Claim 2: All utilities in $\Gamma_{*}$ are zero.

Proof of Claim 2:

Assume in negation that not all utilities in $\Gamma_{*}$ are zero. By the induction hypothesis there exists a mixed-strategy equilibrium $\left(q^{1}, q^{2}\right) \in N\left(\Gamma_{*}\right)$ such that

$$
u\left(q^{1}, q^{2}\right)>0 .
$$

Extend $q^{1}$ to the mixed strategy $r^{1}$ of player 1 in $\Gamma$ by defining $r^{1}\left(s^{1}\right)=0$. Obviously, $u^{i}\left(r^{1}, q^{2}\right)=u^{i}\left(q^{1}, q^{2}\right)$ for $i=1,2$ and therefore (18) implies

$$
u\left(r^{1}, q^{2}\right)>0 .
$$

Since $v_{N}(\Gamma)=0,(19)$ implies that $\left(r^{1}, q^{2}\right)$ is not a mixed-strategy equilibrium in $\Gamma$. However, since $r^{1}$ coincide with $q^{1}$ on $T^{1}, r^{1}\left(s^{1}\right)=0$, and $\left(q^{1}, q^{2}\right)$ is a mixed-strategy equilibrium in $\Gamma_{*}$, player 2 does not have a profitable deviation from $q^{2}$ in $\Gamma$ when player 1 uses $r^{1}$. Therefore, player 1 must have a profitable deviation from $r^{1}$ in $\Gamma$ when player 2 uses $q^{2}$. In particular, player 1 must have a pure-strategy profitable deviation. Since $\left(q^{1}, q^{2}\right)$ is a mixed-strategy equilibrium in $\Gamma_{*}$, the only potential pure-strategy profitable deviation of player 1 in $\Gamma$ is $s_{1}$. Therefore,

$$
u^{1}\left(s^{1}, q^{2}\right)>u^{1}\left(r^{1}, q^{2}\right)
$$

which implies in particular that

$$
u^{1}\left(s^{1}, q^{2}\right)>0 .
$$

Next we show that $\left(s^{1}, q^{2}\right) \in N(\Gamma)$. Since $\left(q^{1}, q^{2}\right)$ is a mixed strategy equilibrium in $\Gamma_{*}$, $u^{1}\left(q^{1}, q^{2}\right) \geq u^{1}\left(t^{1}, q^{2}\right)$ for every $t^{1} \in S^{1}, t^{1} \neq s^{1}$. Since $r^{1}\left(s^{1}\right)=0, u^{1}\left(r^{1}, q^{2}\right) \geq u^{1}\left(t^{1}, q^{2}\right)$ for every $t^{1} \in S^{1}, t^{1} \neq s^{1}$. Therefore, by (20), $s^{1}$ is a best-response to $q^{2}$ in $\Gamma$. By (ii) in Claim 1 , every pure-strategy of player 2 is a best-response to $s^{1}$ in $\Gamma$ and hence, every mixedstrategy of player 2 is a best response to $s^{1}$, and in particular so is $q^{2}$. Therefore, $\left(s^{1}, q^{2}\right)$ is indeed a mixed-strategy equilibrium in $\Gamma$. Since $v_{N}(\Gamma)=0, u^{1}\left(s^{1}, r^{2}\right)=0$, contradicting (21). This complete the proof of Claim 2.

By Claim 2 and by (ii) in Claim $1,\left(s^{1}, t^{2}\right)$ is in $N(\Gamma)$ for every $t^{2} \in S^{2}$. Since $v_{N}(\Gamma)=0$, $u\left(s^{1}, t^{2}\right)=0$ for every $t^{2} \in S^{2}$. Hence, for $i=1,2, u^{i}\left(s^{1}, t^{2}\right)=0$ for every $t^{2} \in S^{2}$. As each $u^{i}$ is identically zero in $\Gamma_{*}$, it follows that for $i=1,2, u^{i}\left(t^{1}, t^{2}\right)=0$ for every $t^{1} \in S^{1}$ and for every $t^{2} \in S^{2}$.

\subsection{The Enforcement Value}

The next theorem shows that the enforcement value is unbounded even in classes of small games.

Theorem $4 E V\left(\mathcal{G}_{m_{1} \times \cdots \times m_{n}}\right)=\infty$ for every $n \geq 2$ and for every $m_{1}, m_{2} \geq 2$.

Proof: By Lemma 2 it suffices to prove the theorem for the case $n=2, m_{1}=2$, and $m_{2}=2$. Consider the following parametric Prisoners' Dilemma game $\Gamma_{x}, x>1$, given in Figure 5: 


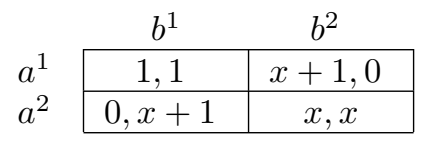

Figure 5

By Lemma 1, every such game has a unique correlated equilibrium $\left(a^{1}, b^{1}\right)$ whose welfare is 2 . However for every $x \geq 1$, opt $\left(\Gamma_{x}\right)=2 x$. Therefore $E V\left(\Gamma_{x}\right) \rightarrow \infty$ when $x \rightarrow \infty$.

The proof of Theorem 4 is based on constructing Prisoner's Dilemma games with a parameter $x$. In particular every player has a weakly dominant strategy in each one of these games. The next theorem shows that even when ruling out weakly dominant strategies, the enforcement value is unbounded.

Theorem $5 E V\left(\left\{\Gamma \mid \Gamma \in \mathcal{G}_{2 \times 2 \times 2}\right.\right.$, no player has a weakly dominant strategy $\}=\infty$.

Proof: Consider the family of parametric games $\Gamma_{z, \epsilon}$ (Figure 6), where $z=\frac{1}{\epsilon^{2}}$

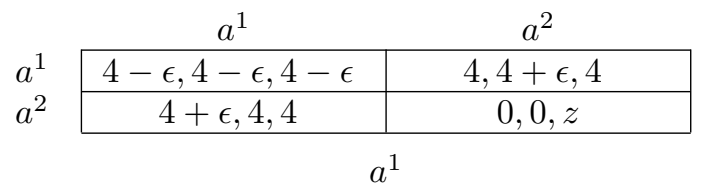

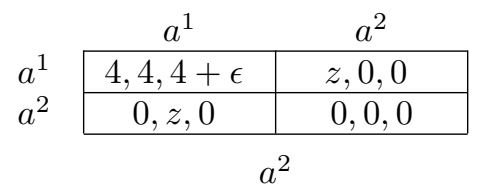

Figure 6

First observe that $\operatorname{opt}\left(\Gamma_{z, \epsilon}\right)=z$ for every $0<\epsilon \leq 0.25$. In order to prove the result we use the dual program $\widehat{D}$. By the weak duality theorem every feasible solution $(\alpha, \beta)$ for the dual problem $\widehat{D}$ satisfies $\beta \geq v_{C}(\Gamma)$. Let $x_{1}, x_{2}, x_{3}$ denote $\alpha^{1}\left(a_{1} \mid a_{2}\right), \alpha^{2}\left(a_{1} \mid a_{2}\right)$ and $\alpha^{3}\left(a_{1} \mid a_{2}\right)$ respectively. Let $y_{1}, y_{2}, y_{3}$ denote $\alpha^{1}\left(a_{2} \mid a_{1}\right), \alpha^{2}\left(a_{2} \mid a_{1}\right)$ and $\alpha^{3}\left(a_{2} \mid a_{1}\right)$ respectively. The dual constraints can be written in the following way (recall that $\left.z=\frac{1}{\epsilon^{2}}\right)$ :

$$
\begin{array}{r}
2 \epsilon y_{1}+2 \epsilon y_{2}+2 \epsilon y_{3}+\beta \geq 12-3 \epsilon, \\
-4 y_{1}-4 y_{3}-2 \epsilon x_{2}+\beta \geq 12+\epsilon, \\
-4 y_{2}-4 y_{3}-2 \epsilon x_{1}+\beta \geq 12+\epsilon, \\
-4 y_{1}-4 y_{2}-2 \epsilon x_{3}+\beta \geq 12+\epsilon, \\
-\frac{1}{\epsilon^{2}} y_{1}+4 x_{2}+4 x_{3}+\beta \geq \frac{1}{\epsilon^{2}}, \\
-\frac{1}{\epsilon^{2}} y_{2}+4 x_{1}+4 x_{3}+\beta \geq \frac{1}{\epsilon^{2}}, \\
-\frac{1}{\epsilon^{2}} y_{3}+4 x_{1}+4 x_{2}+\beta \geq \frac{1}{\epsilon^{2}}, \\
\frac{1}{\epsilon^{2}} x_{1}+\frac{1}{\epsilon^{2}} x_{2}+\frac{1}{\epsilon^{2}} x_{3}+\beta \geq 0 .
\end{array}
$$

Set $y_{1}=y_{2}=y_{3}=x_{1}=0, \beta=\frac{1}{\epsilon}$, and $x_{2}=x_{3}=\frac{1}{4 \epsilon^{2}}$, and observe that it is a feasible solution for every sufficiently small $\epsilon>0$. Note that $E V\left(\Gamma_{z, \epsilon}\right) \geq \frac{z}{\beta}$. However $\frac{z}{\beta}=\frac{1}{\epsilon} \rightarrow \infty$ as $\epsilon \rightarrow 0$, which completes the proof. 


\section{Simple Congestion Games}

In this section we explore the mediation and enforcement values in simple congestion games. We first need a few notations and definitions.

A simple congestion form $F=\left(N, M,\left(w_{j}\right)_{j \in M}\right)$ is defined as follows. $N$ is a nonempty set of players; we keep our assumption that, whenever is convenient, $N=\{1,2, \ldots, n\}$, where $n=|N|$ is the number of players. $M$ is a nonempty set of facilities; Unless otherwise specified we assume $M=\{1,2, \ldots, m\}$. For $j \in M$, let $w_{j} \in \mathbb{R}^{n}$ be the facility payoff function, where for every $1 \leq k \leq n, w_{j}(k)$ denotes the payoff of each user of facility $j$, if there are exactly $k$ users. A congestion form is nonnegative if for every $j \in M, w_{j}$ is nonnegative. Let $\mathcal{Q}$ be the class of all nonnegative simple congestion forms and denote by $\mathcal{Q}_{n \times m} \subseteq \mathcal{Q}$ the class of all nonnegative simple congestion forms with $n$ players and $m$ facilities. Every simple congestion form $F=\left(N, M,\left(w_{j}\right)_{j \in M}\right)$ defines a simple congestion game $\Gamma_{F}=\left(N,\left(S^{i}\right)_{i \in N},\left(u^{i}\right)_{i \in N}\right)$, where $N$ is the set of players, $S^{i}=M$ for every player $i \in N$, and the utility functions $\left(u^{i}\right)_{i \in N}$ are defined as follows.

Let $\mathbf{S}=\times_{i \in N} S^{i}$. For every $\mathbf{A}=\left(A^{1}, A^{2}, \ldots, A^{n}\right) \in \mathbf{S}$ and every $j \in M$, let $\sigma_{j}(\mathbf{A})=$ $\left|\left\{i \in N: A^{i}=j\right\}\right|$ be the number of users of facility $j$. Define $u^{i}: \mathbf{S} \rightarrow \mathbb{R}$ by

$$
u^{i}(\mathbf{A})=w_{A^{i}}\left(\sigma_{A^{i}}(\mathbf{A})\right)
$$

We say that a facility $j$ is non-increasing if $w_{j}(k)$ is a non-increasing function of $k$. Define $\mathcal{Q N}_{n \times m} \subseteq \mathcal{Q}_{n \times m}$ as follows:

$$
\mathcal{Q N}_{n \times m} \triangleq\left\{F \in \mathcal{Q}_{n \times m} \mid \text { all facilities in F are non-increasing }\right\}
$$

We will call a facility $j$ linear if there exist a constant $d_{j}$ such that $w_{j}(k+1)-w_{j}(k)=d_{j}$ for every $k \geq 1$, that is $w_{j}(k)=d_{j} k+\delta_{j}$ for every $k \geq 1$, where $\delta_{j}$ is a constant.

Let $F$ be a simple congestion form with $n$ players and $m$ facilities. A congestion vector $\pi=\pi(n, m)$ is an $m$-tuple $\pi=\left(\pi_{j}\right)_{j \in M}$, where $\pi_{1}, \pi_{2}, \ldots, \pi_{m} \in Z^{*}$ (the set of nonnegative integers) and $\sum_{j=1}^{m} \pi_{j}=n$. $\pi$ represents the situation in which $\pi_{j}$ players choose facility $j$. Every strategy profile $\mathbf{A} \in \mathbf{S}$ uniquely determines a congestion vector $\pi^{\mathbf{A}}$. Note that there are $\left(\begin{array}{c}n \\ \pi_{1}\end{array}\right)\left(\begin{array}{c}n-\pi_{1} \\ \pi_{2}\end{array}\right) \cdots\left(\begin{array}{c}n-\sum_{j=1}^{m-2} \pi_{j} \\ \pi_{m-1}\end{array}\right)$ strategy profiles in the game $\Gamma_{F}$ that correspond to a congestion vector $\pi$, and denote by $B_{\pi}$ the set of all such strategy profiles. Thus $B_{\pi}=$ $\left\{\mathbf{A} \in \mathbf{S} \mid \pi^{\mathbf{A}}=\pi\right\}$. Given a congestion vector $\pi$, all strategy profiles in $B_{\pi}$ have the same welfare which we denote by $u(\pi)$. That is $u(\pi)=\sum_{\left\{j \in M \mid \pi_{j}>0\right\}} \pi_{j} w_{j}\left(\pi_{j}\right)$.

We say that a congestion vector $\pi$ is in equilibrium if every strategy profile in $B_{\pi}$ is a purestrategy equilibrium. A congestion form is called facility symmetric if $w_{j}=w_{k} \quad \forall j, k \in M$. Obviously, a facility symmetric congestion form induces a symmetric game in strategic form. Let $\mathcal{I}_{n \times m} \subseteq \mathcal{Q}_{n \times m}$ be defined by

$$
\mathcal{I}_{n \times m}=\left\{F \in \mathcal{Q}_{n \times m} \mid \text { F is facility symmetric }\right\} \text {. }
$$

Define $\mathcal{I N}_{n \times m} \subseteq \mathcal{I}_{n \times m}$ as follows

$$
\mathcal{I N}_{n \times m}=\left\{F \in \mathcal{I}_{n \times m} \mid \text { all facilities in } \mathrm{F} \text { are non-increasing }\right\} .
$$




\subsection{The Mediation Value}

Although congestion games are especially interesting when the number of players is large, we begin with presenting results for the case in which we have only two players, extending upon the results in the previous section. Following that, we consider the more general $n$-player case.

\subsubsection{The TWO-PlayeR CASE $(n=2)$}

Theorem 1 provides a $\frac{4}{3}$ a tight upper bound for the mediation value in the class of games $\mathcal{G}_{2 \times 2}$. Hence, $\frac{4}{3}$ is obviously an upper bound for the mediation value of nonnegative simple congestion games with two players and two facilities, i.e., games generated by congestion forms in $\mathcal{Q}_{2 \times 2}$. It turns out that this bound is tight:

Theorem $6 M V\left(\left\{\Gamma_{F} \mid F \in \mathcal{Q}_{2 \times 2}\right\}\right)=\frac{4}{3}$.

Proof: Let $F_{x}, x>1$, be the following simple congestion form: $M=\left\{a_{1}, a_{2}\right\}, w_{a_{1}}=(x, 0)$, and $w_{a_{2}}=(1, x-1)$. The games $\Gamma_{F_{x}}, x>1$ are obtained from the games, $\Gamma_{x}$ defined in the proof of Theorem 1 (See Figure 2) by renaming the strategies of player 2. Hence, by what is proved in Theorem $1, M V\left(\Gamma_{F_{x}}\right) \rightarrow \frac{4}{3}$ as $x \rightarrow \infty$.

Next we consider the more general case in which two players can choose among $m$ facilities:

Theorem $7 M V\left(\left\{\Gamma_{F} \mid F \in \mathcal{Q}_{2 \times m}\right\}\right) \leq 2$ for every $m \geq 2$.

Proof: Let $F \in \mathcal{Q}_{2 \times m}$. By Rosenthal (1973) there exists a pure-strategy equilibrium in $\Gamma_{F}$. Let $\mathbf{A}$ be a fixed pure-strategy equilibrium with the largest welfare, that is

$$
u(\mathbf{A}) \geq u(\mathbf{D}) \quad \text { for every pure-strategy equilibrium } \mathbf{D} \text {. }
$$

Let $j$ and $k$ be the facilities that players 1 and 2 choose in $\mathbf{A}$ respectively. We prove by separation to two cases, $j=k$ and $j \neq k$.

Case 1: $j=k$. We prove the theorem in this case by separation to cases $w_{j}(1)>w_{j}(2)$ and $w_{j}(1) \leq w_{j}(2)$.

Case 1.1: $w_{j}(1)>w_{j}(2)$. In this case we show that $j$ is a strictly dominant strategy, which implies by Lemma 1 that $M V\left(\Gamma_{F}\right)=1$. Indeed, let $h \neq j$ be a facility. In order to prove that $j$ strictly dominates $h$ we have to show that the following three inequalities hold: $w_{h}(1)<w_{j}(2), w_{h}(2)<w_{j}(1)$, and $w_{h}(1)<w_{j}(1)$. However, since $w_{j}(1)>w_{j}(2)$, only the first two inequalities should be proved. We first prove that $w_{h}(1)<w_{j}(2)$ : Since A is a pure strategy equilibrium in which both players choose $j, w_{h}(1) \leq w_{j}(2)$. Suppose in negation that $w_{h}(1)=w_{j}(2)$. Since $\mathbf{A}$ is a pure-strategy equilibrium, $w_{j}(2) \geq w_{l}(1)$ for every $l \neq j$, and therefore, $w_{h}(1) \geq w_{l}(1)$ for every $l \neq j$. If, in addition, $w_{j}(1) \geq w_{h}(2)$, the strategy profile in which one player chooses $h$, and the other player chooses $j$ is a purestrategy equilibrium and obtains a larger welfare than A contradicting (23). Therefore, $w_{h}(2)>w_{j}(1)$. This implies that the strategy profile in which both players choose $h$ is a pure-strategy equilibrium and obtains a larger welfare than $\mathbf{A}$, again a contradiction to (23). Therefore $w_{h}(1)<w_{j}(2)$. 
We next show that $w_{h}(2)<w_{j}(1)$. If $w_{h}(2) \geq w_{j}(1)$ the strategy profile in which both players choose $h$ is a pure-strategy equilibrium and $2 w_{h}(2) \geq 2 w_{j}(1)>2 w_{j}(2)$ contradicting (23). This completes the proof in Case 1.1.

Case 1.2: $w_{j}(1) \leq w_{j}(2)$. Let $\mathbf{B}$ be a pure strategy profile in which the maximum welfare is obtained. That is, $u(\mathbf{B})=\operatorname{opt}\left(\Gamma_{F}\right)$. In order to prove the theorem it suffices to prove that

$$
u(\mathbf{B}) \leq 2 u(\mathbf{A}) .
$$

Suppose in B the players choose distinct facilities (one of these facilities can be $j$ ). Since $\mathbf{A}$ is a pure-strategy equilibrium, $w_{l}(1) \leq w_{j}(2)$ for every $l \neq j$. Therefore, since also $w_{j}(1) \leq w_{j}(2), u(\mathbf{B}) \leq u(\mathbf{A})$ and in particular (24) holds. Suppose the two players choose the same facility in $\mathbf{B}$, and denote this facility by $s$. If $s=j \mathbf{B}=\mathbf{A}$ and (24) holds. Therefore we assume $s \neq j$. If $w_{s}(2) \leq w_{j}(2), u(\mathbf{B}) \leq u(\mathbf{A})$, which implies (24). Therefore we can assume $w_{s}(2)>w_{j}(2)$ and in particular $w_{s}(2)>w_{j}(1)$. Since $w_{j}(2) \geq w_{l}(1)$ for every facility $l, l \neq j, \mathbf{B}$ is a pure-strategy equilibrium and $u(\mathbf{B})>u(\mathbf{A})$, contradicting (23). Hence the proof of Case 1.2 is complete.

Case 2: $j \neq k$. Let $\mathbf{B}$ be an arbitrary pure strategy profile in which the maximum welfare is obtained. That is, $u(\mathbf{B})=\operatorname{opt}\left(\Gamma_{F}\right)$. It suffices to prove that $(24)$ holds.

Recall that $\mathbf{A}$ is a pure-strategy equilibrium. If in $\mathbf{B}$ each player chooses a different facility, $u(\mathbf{B}) \leq u(\mathbf{A})$ and in particular $(24)$ holds. Therefore we assume that in $\mathbf{B}$ both players choose the same facility $l(l$ can be $j$ or $k$ ). We claim that

$$
w_{l}(2) \leq \max \left\{w_{j}(1), w_{k}(1)\right\}
$$

Indeed, if (25) does not hold, $\mathbf{B}$ is a pure strategy equilibrium, because a player in $\mathbf{B}$ does not want to deviate to either $j$ or $k$, and she does not want to deviate to any other facility because $\mathbf{A}$ is a pure strategy equilibrium. Since $u(\mathbf{B})>u(\mathbf{A})$ this contradicts (23). Since $u(\mathbf{B})=2 w_{l}(2)$ and $\max \left\{w_{j}(1), w_{k}(1)\right\} \leq u(\mathbf{A}),(25)$ implies $(24)$.

Notice that Theorems 6 and 7 imply that correlation can help in congestion games with only two players. the next theorem shows that correlation cannot help increasing social welfare when the facilities are non-increasing:

Theorem $8 M V\left(\mathcal{Q N}_{2 \times m}\right)=1$ for every $m \geq 2$.

Proof: Let $F \in \mathcal{Q N}_{2 \times m}$. Let $j \in M$ be such that $w_{j}(1) \geq w_{l}(1)$ for all $l \in M$. If $w_{j}(2)>w_{l}(1)$ for all $l \neq j, j$ is a strictly dominant strategy, which implies by Lemma 1 that $M V\left(\Gamma_{F}\right)=1$. Suppose there exist some facility $k, k \neq j$ such that $w_{j}(2) \leq w_{k}(1)$. Choose such $k$ for which $w_{k}(1)$ is maximal, that is $w_{l}(1) \leq w_{k}(1)$ for every $l, l \neq j$. Therefore, the strategy profile in which one player chooses $j$ and the other player chooses $k$ is a pure strategy equilibrium which obtains the maximal welfare. The existence of such a strategy profile implies that $M V\left(\Gamma_{F}\right)=1 . \square$

\subsubsection{Simple CONGESTION GAMES With $n$ PlAyeRS}

Theorems 2 and 3 in Section 3.1.2 imply that correlation has an unbounded value when considering arbitrary games. The next theorem shows that correlation has similar effects in the context of simple congestion games. In particular, we show that the mediation value is unbounded with the presence of an additional player: 
Theorem $9 M V\left(\left\{\Gamma_{F} \mid F \in \mathcal{Q N}_{3 \times 2}\right\}\right)=\infty$.

Proof: Consider the following family of forms $F_{\epsilon}, 0<\epsilon \leq 0.5: M=\left\{a_{1}, a_{2}\right\}, w_{a_{1}}=$ $(z, 4,4-\epsilon), w_{a_{2}}=(4+\epsilon, 0,0)$, where $z=\frac{1}{\epsilon^{2}}$. Observe that the games, $\Gamma_{F_{\epsilon}}$ are the games given in the proof of Theorem 5 (Figure 6 ), and that the monotonicity condition is satisfied for every $0<\epsilon \leq 0.5$.

We first show that for every sufficiently small $\epsilon$, the welfare in every mixed-strategy equilibrium is lower than 13. After that we will construct, for every sufficiently small $\epsilon$, a correlated equilibria in $\Gamma_{F_{\epsilon}}$ such that the welfare in these correlated equilibria approaches infinity as $\epsilon \rightarrow 0$.

Note that the only pure-strategy equilibria are the strategy profiles in which two players play $a^{1}$ and one player plays $a^{2}$. The welfare in each of these strategy profiles is $12+\epsilon$, which is less than 13.

We proceed to deal with mixed-strategy equilibria in which at least one player does not play a pure strategy. In such an equilibrium, no player plays the strategy $a^{2}$ with probability one because if one of the players does it, the only mixed-strategy equilibria are those in which each of the other two players plays $a^{1}$ with probability one, i.e., the equilibrium is pure.

Suppose some player plays $a^{1}$ with probability one. Note that the utility matrix of the other two players is as given in Figure 7 .

\begin{tabular}{l|c|c|} 
& \multicolumn{1}{c}{$a^{1}$} & $a^{2}$ \\
\cline { 2 - 3 }$a^{1}$ & $4-\epsilon, 4-\epsilon$ & $4,4+\epsilon$ \\
\cline { 2 - 3 }$a^{2}$ & $4+\epsilon, 4$ & 0,0 \\
\cline { 2 - 3 } & &
\end{tabular}

Figure 7

Therefore it is standard to check that there exists a unique mixed-strategy equilibrium in which one of the players play $a^{1}$ with probability 1 , and that this equilibrium, when this player is player 1 is:

$$
\left((1,0),\left(\frac{2}{2+\epsilon}, \frac{\epsilon}{2+\epsilon}\right),\left(\frac{2}{2+\epsilon}, \frac{\epsilon}{2+\epsilon}\right)\right) .
$$

Since the game is symmetric, the only mixed-strategy equilibria are the three permutations of this vector. The welfare in each of these mixed-strategy equilibria is

$$
\begin{gathered}
3(4-\epsilon)\left(\frac{2}{2+\epsilon}\right)^{2}+2(12+\epsilon) \cdot \frac{2 \epsilon}{(2+\epsilon)^{2}}+z \cdot\left(\frac{\epsilon}{2+\epsilon}\right)^{2}= \\
\frac{48+36 \epsilon+(z+4) \epsilon^{2}}{(2+\epsilon)^{2}} \rightarrow 12.25
\end{gathered}
$$

as $\epsilon \rightarrow 0$.

Consider a completely mixed-strategy equilibrium. That is, every player assigns positive probabilities to both facilities. Denote this equilibrium by $((p, 1-p),(q, 1-q),(h, 1-$ h)) $0<p, q, h<1$. Because player 1 is indifferent between $a^{1}$ and $a^{2}$ given that players 2 and 3 play $(q, 1-q)(h, 1-h)$ respectively,

$$
(4-\epsilon) q h+4(1-q) h+4(1-h) q+z(1-h)(1-q)=(4+\epsilon) q h .
$$


Note that similar equalities hold for players 2 and 3 , when $q$ and $h$ are exchanged with $p$ respectively. For every fixed $h$ there exists a unique $q$ that solves equation (26). Therefore, by permuting the names of the players, $p=q=h$. This enables us to reduce Equation (26) to

$$
(z-8-2 \epsilon) p^{2}+(8-2 z) p+z=0
$$

which yields

$$
p=\frac{2 z-8-\sqrt{64+8 z \epsilon}}{2 z-16-4 \epsilon}
$$

because the other solution of (28) does not satisfy $0<p<1$. Therefore, the welfare in this completely mixed-strategy equilibrium is

$$
\begin{gathered}
3 p(1-p)^{2} z+3 p^{2}(1-p)(12+\epsilon)+p^{3}(12-3 \epsilon)= \\
(3 z-24-5 \epsilon) p^{3}-(6 z-36+3 \epsilon) p^{2}+3 z p .
\end{gathered}
$$

Let $A=(36-3 \epsilon) p^{2}-(24+5 \epsilon) p^{3}$ and $B=3 z p^{3}-6 z p^{2}+3 z p$. Hence $(29)=A+B$. We show that $A \rightarrow 12$ and $B \rightarrow 0$ as $\epsilon \rightarrow 0$. Observe that $p \rightarrow 1$ as $\epsilon \rightarrow 0$. This implies that $A \rightarrow 12$ as $\epsilon \rightarrow 0$. Observe that $z \epsilon=\sqrt{z}$. Thus, from (28) and for small enough $\epsilon$ we have that $p \approx \hat{p}=\frac{2 z-\sqrt{8} z^{\frac{1}{4}}}{2 z}=1-\frac{\sqrt{8}}{2} z^{-\frac{3}{4}}$. For simplicity set $c=\frac{\sqrt{8}}{2}$. Therefore

$$
\begin{gathered}
B \approx 3 z\left(1-c z^{-\frac{3}{4}}\right)^{3}-6 z\left(1-c z^{-\frac{3}{4}}\right)^{2}+3 z\left(1-c z^{-\frac{3}{4}}\right)= \\
3 z\left[1-3 c z^{-\frac{3}{4}}+3 c^{2} z^{-\frac{6}{4}}-c^{3} z^{-\frac{9}{4}}\right]-6 z\left[1-2 c z^{-\frac{3}{4}}+c^{2} z^{-\frac{6}{4}}\right]+3 z\left(1-c z^{-\frac{3}{4}}\right)= \\
3 c^{2} z^{-\frac{2}{4}}-3 c^{3} z^{-\frac{5}{4}}= \\
3 c^{2} \epsilon-3 c^{3} \epsilon^{\frac{10}{4}} \rightarrow 0
\end{gathered}
$$

as $\epsilon \rightarrow 0$.

This completes the proof that $v_{N}\left(\Gamma_{F_{\epsilon}}\right) \leq 13$ for every sufficiently small enough $\epsilon$.

To complete the proof of the theorem we construct, for every sufficiently small $\epsilon$, a correlated equilibria in $\Gamma_{F_{\epsilon}}$ such that the welfare in these correlated equilibria approaches infinity as $\epsilon \rightarrow 0$.

In order that a correlated strategy $\mu$ will be a correlated equilibrium in $\Gamma_{F_{\epsilon}}$, the following inequalities should be satisfied (in brackets we relate the inequality to the corresponding inequality in $(2)):^{14}$

$$
\begin{aligned}
& \text { 1. }-2 \epsilon \mu(1,1,1)+4 \mu(1,2,1)+4 \mu(1,1,2)+z \mu(1,2,2) \geq 0 . \quad\left(i=1, s^{i}=a^{1}, t^{i}=a^{2}\right) \\
& \text { 2. } 2 \epsilon \mu(2,1,1)-4 \mu(2,2,1)-4 \mu(2,1,2)-z \mu(2,2,2) \geq 0 . \quad\left(i=1, s^{i}=a^{2}, t^{i}=a^{1}\right) \\
& \text { 3. }-2 \epsilon+4 \mu(2,1,1)+4 \mu(1,1,2)+z \mu(2,1,2) \geq 0 . \quad\left(i=2, s^{i}=a^{1}, t^{i}=a^{2}\right) \\
& \text { 4. } 2 \epsilon \mu(1,2,1)-4 \mu(2,2,1)-4 \mu(1,2,2)-z \mu(2,2,2) \geq 0 . \quad\left(i=2, s^{i}=a^{2}, t^{i}=a^{1}\right) \\
& \text { 5. }-2 \epsilon \mu(1,1,1)+4 \mu(1,2,1)+4 \mu(2,1,1)+z \mu(2,2,1) \geq 0 . \quad\left(i=3, s^{i}=a^{1}, t^{i}=a^{2}\right)
\end{aligned}
$$

14. Here, we slightly abuse notation by letting $\mu(i, j, k)=\mu\left(a^{i}, a^{j}, a^{k}\right)$. 
6. $2 \epsilon \mu(1,1,2)-4 \mu(1,2,2)-4 \mu(2,1,2)-z \mu(2,2,2) \geq 0 . \quad\left(i=3, s^{i}=a^{2}, t^{i}=a^{1}\right)$

7. $\sum_{i=1}^{8} \mu_{i}=1$.

For sufficiently small $\epsilon$, the above inequalities are satisfied by $\mu_{\epsilon}$, where $\mu_{\epsilon}(1,1,1)=$ $\mu_{\epsilon}(2,1,2)=\mu_{\epsilon}(2,2,2)=0, \mu_{\epsilon}(2,1,1)=\mu_{\epsilon}(1,1,2)=\epsilon^{\frac{1}{4}}$, and $\mu_{\epsilon}(2,2,1)=\mu_{\epsilon}(1,2,2)=\epsilon^{\frac{3}{2}}$.

Note that $z \mu(2,2,1)=\frac{1}{\sqrt{\epsilon}} \rightarrow \infty$ as $\epsilon \rightarrow 0$, and that $u(\mu) \geq z \mu(2,2,1)$. Therefore $u(\mu) \rightarrow \infty$ as $\epsilon \rightarrow 0$.

We conjecture that Theorem 9 holds for more players and/or more facilities. That is, $M V\left(\left\{\Gamma_{F} \mid F \in \mathcal{Q N}_{n \times m}\right\}\right)=\infty$ for every $n \geq 3$ and for every $m \geq 2$. One way to prove this conjecture is to modify Lemma 2, which is used to prove analogous extensions for general games (see e.g., Theorems 2 and 3).

The next theorem deals with linear facilities:

\section{Theorem 10}

$$
M V\left(\left\{\Gamma_{F} \mid F \in \mathcal{Q N}_{n \times 2}, \text { all facilities of } F \text { are linear }\right\}\right) \leq \phi
$$

for every $n \geq 2$, where

$$
\phi=(\sqrt{5}+1) / 2 .
$$

The following lemma (Schrijver, 1986, page 61) is used in the proof of Theorem 10.

Lemma 5 (Farkas Lemma) Let $s, t$ be positive integers. Given a matrix A of dimensions $s \times t$ and a vector $\mathbf{b} \in \mathbb{R}^{s}$, one and only one of the following systems has a solution:

$$
\begin{array}{cl}
\text { (i) } & \mathbf{A x} \geq \mathbf{b}, \quad \mathbf{x} \in \mathbb{R}^{t} ; \\
\text { (ii) } & \mathbf{y}^{T} \mathbf{A}=0, \quad \mathbf{y}^{T} \mathbf{b}>0, \quad \mathbf{y} \in \mathbb{R}_{+}^{s},
\end{array}
$$

where $\mathbb{R}_{+}^{s}$ denotes the set of all nonnegative vectors in $\mathbb{R}^{s}$.

Proof of Theorem 10: Consider the nonnegative, non-increasing and linear congestion form in which $M=\{f, g\}$, and $w_{f}$ and $w_{g}$ are the facility payoff functions of $f$ and $g$ respectively, where $w_{f}(k)=d_{f} k+\delta_{f}$ and $w_{g}(k)=d_{g} k+\delta_{g}$. Obviously, $d_{f}, d_{g} \leq 0$. Denote by $\Gamma$ the induced congestion game. Assume w.l.o.g. that

$$
w_{f}(1) \geq w_{g}(1)
$$

Denote by $\pi_{k}=(n-k, k)$ the congestion vector in which $n-k$ players choose $f$ and $k$ players choose $g$. Let $\tau, 0 \leq \tau \leq n$, be the largest integer such that $\pi_{\tau}$ is in equilibrium. Since $\pi_{\tau}$ is in equilibrium, $w_{g}(\tau) \geq w_{g}(n-\tau+1)$, and because of the monotonicity condition,

$$
w_{g}(j) \geq w_{g}(n-j+1) \quad \forall j \leq \tau .
$$

Recall that the welfare in $\pi_{\tau}$ is denoted by $u\left(\pi_{\tau}\right)$.

Claim 1: $M V(\Gamma))=1$, whenever $\tau \in\{n, 0\}$. 
Proof: Suppose $\tau=n$. Since $(0, n)$ is in equilibrium, $w_{g}(n) \geq w_{f}(1)$. Therefore, by $(31)$, $w_{g}(n) \geq w_{g}(1)$. By the monotonicity condition, for every $k, u\left(\pi_{k}\right)=(n-k) w_{f}(n-k)+$ $k w_{g}(k) \leq(n-k) w_{f}(1)+k w_{g}(1)$. Therefore, $u\left(\pi_{k}\right) \leq n w_{g}(n)=u\left(\pi_{n}\right)$. Hence, the maximal welfare, $\operatorname{opt}(\Gamma)$ is attained at the equilibrium $\pi_{n}$, which implies that the mediation value equals 1 .

Suppose $\tau=0$. Since $(n, 0)$ is an equilibrium, $w_{f}(n) \geq w_{g}(1)$. We claim that

$$
w_{f}(n)>w_{g}(1) .
$$

Indeed, assume in negation that $w_{f}(n)=w_{g}(1)$. Since $(n-1,1)$ is not in equilibrium, either $w_{f}(n-1)<w_{g}(2)$ or $w_{g}(1)<w_{f}(n)$. Therefore, $w_{f}(n-1)<w_{g}(2)$, and because of the monotonicity condition, $w_{f}(n)<w_{g}(1)$, which yields a contradiction. Therefore (33) holds, which implies that choosing $f$ strictly dominates choosing $g$ in $\Gamma$. Therefore, by Lemma 1 , every correlated equilibrium in $\Gamma$ is a pure strategy profile, which generates the congestion vector $\pi_{0}$. Hence, the mediation value equals 1.

By Claim 1, at the rest of this proof we assume w.l.og. that

$$
1 \leq \tau \leq n-1
$$

Note that if $w_{g}(1)<w_{f}(n)$, choosing $f$ strictly dominates choosing $g$, which implies that the mediation value equals 1 . Therefore we assume w.l.o.g. at the rest of the proof that

$$
w_{g}(1) \geq w_{f}(n)
$$

The following is a key claim for our proof.

Claim 2: $u\left(\pi_{j}\right) \leq u\left(\pi_{\tau}\right)$ for every $j \leq \tau$.

Proof: Let $j<\tau$. Since the facilities are linear, $w_{g}(j)=w_{g}(\tau)+(\tau-j) d_{g}$ and $w_{f}(n-j)=$ $w_{f}(n-\tau)-(\tau-j) d_{f}$. Hence,

$$
\begin{gathered}
u\left(\pi_{\tau}\right)-u\left(\pi_{j}\right)= \\
s w_{g}(\tau)+(n-\tau) w_{f}(n-\tau)-j\left(w_{g}(\tau)+(\tau-j) d_{g}\right)-(n-j)\left(w_{f}(n-\tau)-(\tau-j) d_{f}\right)= \\
w_{f}(n-\tau)(j-\tau)+w_{g}(\tau)(\tau-j)-j(\tau-j) d_{g}+(n-j)(\tau-j) d_{f}= \\
(\tau-j)\left(w_{g}(\tau)-w_{f}(n-\tau)+(n-j) d_{f}-j d_{g}\right) \geq \\
(\tau-j)\left(w_{f}(n-\tau+1)-w_{f}(n-\tau)+(n-j) d_{f}-j d_{g}\right)
\end{gathered}
$$

where the last inequality follows since $\pi_{\tau}$ is in equilibrium. Since the facilities are linear,

$$
\begin{gathered}
\operatorname{RHS}(36)=(\tau-j)\left((n-j-1) d_{f}-j d_{g}\right)= \\
(\tau-j)\left(w_{f}(1)-w_{f}(n-j)-w_{g}(1)+w_{g}(j+1)\right) .
\end{gathered}
$$

Since $j<\tau$ it suffices to show that $\left(w_{f}(1)-w_{f}(n-j)-w_{g}(1)+w_{g}(j+1)\right) \geq 0$. This inequality follows from (31) and (32).

Next we define an auxiliary strategy profile. Denote by $\tilde{\mathbf{q}}$ the mixed-strategy profile in which players $1,2, \ldots, n-\tau-1$ choose $f$ with probability 1 , and each of the other $\tau+1$ players choose $g$ with probability $\tilde{p}=\frac{w_{g}(1)-w_{f}(n)}{\tau\left(d_{f}+d_{g}\right)}$, i.e., each of them plays the mixed 
strategy $(1-\tilde{p}, \tilde{p})$. By $(35), \tilde{p} \geq 0$. To see that $\tilde{p} \leq 1$, note that because $\pi_{\tau}$ is in equilibrium, $w_{g}(1)-w_{f}(n) \leq w_{g}(1)-w_{g}(\tau+1)+w_{f}(n-\tau)-w_{f}(n)=s d_{g}+s d_{f}$.

Claim 3: (i) $\tilde{\mathbf{q}}$ is a mixed strategy equilibrium; (ii) $u(\tilde{\mathbf{q}})=n w_{f}(n)+\tilde{p} d_{f}((n-\tau-1)(\tau+$ 1) $+(\tau+1) \tau)$.

Proof: (i) We first show that $u^{i}\left(\tilde{\mathbf{q}}^{-i}, f\right) \geq u^{i}\left(\tilde{\mathbf{q}}^{-i}, g\right)$ for every player $i$ that chooses $f$ with probability 1 . Let $k=\tau+1$. We have, $u^{i}\left(\tilde{\mathbf{q}}^{-i}, f\right)-u^{i}\left(\tilde{\mathbf{q}}^{-i}, g\right)=$

$$
\begin{gathered}
\sum_{j=0}^{k}\left(\begin{array}{c}
k \\
j
\end{array}\right) \tilde{p}^{j}(1-\tilde{p})^{k-j}\left(w_{f}(n-j)-w_{g}(j+1)\right)= \\
\sum_{j=0}^{k}\left(\begin{array}{c}
k \\
j
\end{array}\right) \tilde{p}^{j}(1-\tilde{p})^{k-j}\left(w_{f}(1)-(n-j-1) d_{f}-w_{g}(1)+j d_{g}\right)= \\
w_{f}(1)-w_{g}(1)-(n-1) d_{f}+k \tilde{p} d_{f}+k \tilde{p} d_{g} .
\end{gathered}
$$

Since $w_{f}(n)=w_{f}(1)-(n-1) d_{f}$ and $k>k-1$,

$$
R H S(38)=w_{f}(n)-w_{g}(1)+(k-1) \tilde{p} d_{f}+(k-1) \tilde{p} d_{g} \geq 0,
$$

where the last inequality follows since $\tilde{p} \leq 1$, where $\tilde{p}=\frac{w_{g}(1)-w_{f}(n)}{(k-1)\left(d_{f}+d_{g}\right)}$.

We next show that every player $i$ that plays the mixed strategy $(1-\tilde{p}, \tilde{p})$ is indifferent between $f$ and $g$. Observe that $u^{i}\left(\tilde{\mathbf{q}}^{-i}, f\right)-u^{i}\left(\tilde{\mathbf{q}}^{-i}, g\right)=$

$$
\begin{gathered}
\sum_{j=0}^{n}\left(\begin{array}{c}
k-1 \\
j
\end{array}\right) \tilde{p}^{j}(1-\tilde{p})^{k-1-j}\left(w_{f}(n-j)-w_{g}(j+1)\right)= \\
\sum_{j=0}^{n}\left(\begin{array}{c}
k-1 \\
j
\end{array}\right) \tilde{p}^{j}(1-\tilde{p})^{k-1-j}\left(w_{f}(1)-(n-j-1) d_{f}-w_{g}(1)+j d_{g}\right)= \\
w_{f}(1)-w_{g}(1)-(n-1) d_{f}+(k-1) \tilde{p} d_{f}+(k-1) \tilde{p} d_{g}= \\
w_{f}(n)-w_{g}(1)+(k-1) \tilde{p} d_{f}+(k-1) \tilde{p} d_{g}=0 .
\end{gathered}
$$

(ii) Similar calculations as in part (i) yield that the expected payoff for each of the $n-\tau-1$ players that choose $f$ with probability one equals $w_{f}(n)+(\tau+1) \tilde{p} d_{f}$, and that the expected payoff for each of the other $\tau+1$ players equals $w_{f}(n)+\tau \tilde{p} d_{f}$. Therefore $u(\tilde{\mathbf{q}})=n w_{f}(n)+\tilde{p} d_{f}((n-\tau-1)(\tau+1)+(\tau+1) \tau)$.

We proceed with the main proof. Define

$$
Z=\phi \max \left\{u\left(\pi_{\tau}\right), u(\tilde{\mathbf{q}})\right\} .
$$

If $u\left(\pi_{k}\right) \leq Z$ for every $0 \leq k \leq n, \operatorname{opt}(\Gamma) \leq Z$, and therefore, $v_{c}(\Gamma) \leq \operatorname{opt}(\Gamma) \leq$ $\phi \max \left\{u\left(\pi_{\tau}\right), u(\tilde{\mathbf{q}})\right\}$ implying that $M V(\Gamma) \leq \phi$. Therefore we can assume for the rest of the proof that there exist an integer $\hat{k}, 0 \leq \hat{k} \leq n$ such that

$$
u\left(\pi_{\hat{k}}\right)>Z
$$


We proceed by utilizing the dual program $\widehat{D}$. In our case, the dual problem denoted by $\widehat{D}_{\Gamma}$ is as follows:

$$
\widehat{D}_{\Gamma}: \begin{cases}\min \beta & \\ \text { s.t. } & \forall i \in N, \\ \alpha^{i}(f \mid g) \geq 0 & \forall i \in N, \\ \alpha^{i}(g \mid f) \geq 0 & \forall \mathbf{s} \in \mathbf{S}, \\ \sum_{i \in N} \alpha^{i}\left(t^{i} \mid s^{i}\right)\left[u^{i}\left(t^{i}, \mathbf{s}^{-i}\right)-u^{i}\left(\mathbf{s}^{\mathbf{i}}, \mathbf{s}^{-\mathbf{i}}\right)\right]+\beta \geq u(\mathbf{s}) & \end{cases}
$$

where for every $i, t^{i}=f$ if $s^{i}=g$, and $t^{i}=g$ if $s^{i}=f$.

Recall that by the weak duality theorem, for every feasible solution for the dual problem, $(\alpha, \beta), \beta \geq v_{C}(\Gamma)$. Therefore, in order to complete the proof, it suffices to show that there exists a feasible solution for the dual problem $\widehat{D}_{\Gamma}$ in which $\beta=Z$, where $Z$ is defined in (39).

We begin with restricting the range of the variables in the dual problem $(\hat{D})_{\Gamma}$. More specifically, the following system $\left(\widehat{D_{1}}\right)$ is obtained from $(\widehat{D})$ by letting $\beta=Z, \alpha^{i}(g \mid f)=0$ and $x=\alpha^{i}(f \mid g)$ for every $i \in N$, i.e., $x$ remains the only variable:

$$
\widehat{D_{1}}:\left\{\begin{array}{l}
\min \beta \\
\text { s.t. } \\
\beta=Z \\
k\left(w_{f}(n-k+1)-w_{g}(k)\right) x \geq u\left(\pi_{k}\right)-\beta \quad k=1, \ldots, n, \\
x \geq 0 .
\end{array}\right.
$$

Obviously, every optimal solution, $x$ of $\widehat{D_{1}}$ defines a feasible solution, $(\alpha, \beta)$ of $(\hat{D})_{\Gamma}$ in which $\beta=Z$. Since $\beta=Z$, the existence of an optimal solution for $\widehat{D_{1}}$ is equivalent to the existence of a feasible solution for the following system of constraints, $\left(\widehat{D_{2}}\right)$ :

$$
\widehat{D_{2}}:\left\{\begin{array}{l}
k\left(w_{f}(n-k+1)-w_{g}(k)\right) x \geq u\left(\pi_{k}\right)-\beta \quad k=1, \ldots, n \\
x \geq 0
\end{array}\right.
$$

We next show that the inequality $x \geq 0$ in $\widehat{D_{2}}$ is redundant, i.e., that if $x$ satisfies the first $n$ constraints, $x \geq 0$. Recall that by (40) there exist an integer $0 \leq \hat{k} \leq n$ such that $u\left(\pi_{\hat{k}}\right)>Z$. Since $\phi>1$, by Claim $2, \hat{k}>\tau$, and in particular, $\hat{k} \geq 1$. Since $\hat{k}>\tau, \pi_{\hat{k}}$ is not in equilibrium. Since in $\pi_{\tau}$, a player in $f$ does not wish to deviate to $g$, so is the case in $\pi_{\hat{k}}$ in which there are fewer players in $f$. Therefore, in $\pi_{\hat{k}}$, a deviation of a player from $g$ to $f$ is profitable, i.e., $w_{f}(n-\hat{k}+1)-w_{g}(\hat{k})>0$, and since $\hat{k}>0, \hat{k}\left(w_{f}(n-\hat{k}+1)-w_{g}(\hat{k})\right)>0$. Since $x$ satisfies the constraint for $\hat{k}$ in $\widehat{D_{2}}$ and $u\left(\pi_{\hat{k}}\right)>Z=\beta, x>0$. Hence $\widehat{D}_{2}$ is equivalent to

$$
\widehat{D_{3}}:\left\{k\left(w_{f}(n-k+1)-w_{g}(k)\right) x \geq u\left(\pi_{k}\right)-\beta \quad k=1, \ldots, n .\right.
$$


By Farkas Lemma (Lemma 5), $\widehat{D_{3}}$ has a solution $x$ if and only if the following system does not have a solution, $\mathbf{y}=\left(y_{1}, y_{2}, \ldots, y_{n}\right)$ :

$$
\widehat{P_{1}}: \begin{cases}\sum_{k=1}^{n} y_{k} k\left(w_{f}(n-k+1)-w_{g}(k)\right)=0, & \\ \sum_{k=1}^{n} y_{k}\left(u\left(\pi_{k}\right)-\beta\right)>0, & k=1, \ldots, n \\ y_{k} \geq 0 & \end{cases}
$$

Therefore, it suffices to show that $\widehat{P_{1}}$ does not have a solution. Note that if $\mathbf{y}$ is a solution for $\widehat{P_{1}}$, at least one variable $y_{k}$ is positive. Therefore, it suffices to prove that there does not exist a solution for $\widehat{P_{1}}$, which is also a probability vector, i.e., $\sum_{k=1}^{n} y_{k}=1$. Let $\mathbf{y}$ be a probability vector that satisfies the first constraint in $\widehat{P_{1}}$. We have to prove that $\mathbf{y}$ does not satisfy the second constraint, that is, we have to show that

$$
\sum_{k=1}^{n} y_{k} u\left(\pi_{k}\right) \leq \beta
$$

Let $Y$ be the random variable in which $y_{k}=P(Y=k), k=1, \ldots, n$, and recall that the expected value of $Y$ satisfies $E[Y]=\sum_{k=1}^{n} k y_{k}$. We first derive the following useful inequalities given in Claim 4:

\section{Claim 4:}

(i)

$$
E[Y] \leq \frac{w_{g}(1)-w_{f}(n)+d_{f}+d_{g}}{\left(d_{f}+d_{g}\right)}
$$

(ii)

$$
\sum_{k=1}^{n} y_{k} u\left(\pi_{k}\right)=E[Y]\left(w_{f}(1)-w_{f}(n)\right)+n w_{f}(n)
$$

Proof: (i) Since the first constraint in $\widehat{P_{1}}$ is satisfied,

$$
\begin{gathered}
0=\sum_{k=1}^{n} y_{k} k\left(w_{f}(n-k+1)-w_{g}(k)\right)= \\
\sum_{k=1}^{n} y_{k} k\left(w_{f}(n)+(k-1) d_{f}-w_{g}(1)+(k-1) d_{g}\right)= \\
E[Y]\left(w_{f}(n)-w_{g}(1)-d_{f}-d_{g}\right)+E\left(Y^{2}\right)\left(d_{f}+d_{g}\right) .
\end{gathered}
$$

Since $E\left[Y^{2}\right] \geq E[Y]^{2}$,

$$
E[Y]\left(w_{f}(n)-w_{g}(1)-d_{f}-d_{g}\right)+E[Y]^{2}\left(d_{f}+d_{g}\right) \leq 0 .
$$

Since $E[Y]>0$, by dividing both sides by $E[Y]$ we obtain (42). 
(ii) We have

$$
\begin{gathered}
\sum_{k=1}^{n} y_{k} u\left(\pi_{k}\right)=\sum_{k=1}^{n} y_{k}\left(k w_{g}(k)+(n-k) w_{f}(n-k)\right)= \\
\sum_{k=1}^{n} y_{k}\left(k w_{f}(n-k+1)+(n-k) w_{f}(n-k)\right),
\end{gathered}
$$

where the last equality holds because the first constraint in $\widehat{P_{1}}$ is satisfied by y. Therefore,

$$
\begin{gathered}
\sum_{k=1}^{n} y_{k} u\left(\pi_{k}\right)=\sum_{k=1}^{n} y_{k} k\left(w_{f}(1)-(n-k) d_{f}\right)+\sum_{k=1}^{n} y_{k}(n-k) w_{f}(n-k)= \\
w_{f}(1) E[Y]+\sum_{k=1}^{n} y_{k}(n-k)\left(w_{f}(n-k)-k d_{f}\right)=w_{f}(1) E[Y]+w_{f}(n) \sum_{k=1}^{n} y_{k}(n-k) \\
=E[Y]\left(w_{f}(1)-w_{f}(n)\right)+n w_{f}(n),
\end{gathered}
$$

which proves that $(43)$ holds.

We proceed to prove that (41) holds. By plugging (43) in (41), we can equivalently prove that

$$
E[Y]\left(w_{f}(1)-w_{f}(n)\right)+n w_{f}(n) \leq \beta,
$$

and we distinguish between the following two cases:

Case 1: $\tilde{p}<1 / \phi$. First we show that

$$
E[Y] \leq \tau \phi
$$

Because $\tilde{p}<1 / \phi,\left(w_{g}(1)-w_{f}(n)\right) /\left(d_{f}+d_{g}\right) \leq \tau / \phi$. Hence, by $(42), E[Y] \leq \frac{\tau}{\phi}+1$. Since $\phi=\frac{1}{\phi}+1, \tau \phi=\frac{\tau}{\phi}+\tau$. Since $\tau \geq 1$, (45) holds.

Suppose in negation that (44) does not hold, i.e., $E[Y]\left(w_{f}(1)-w_{f}(n)\right)+n w_{f}(n)>\beta$. Note that $\beta=Z \geq \phi u\left(\pi_{\tau}\right)$. Therefore, $E[Y]\left(w_{f}(1)-w_{f}(n)\right)+n w_{f}(n)>\phi u\left(\pi_{\tau}\right)$. By the definition of $u\left(\pi_{\tau}\right)$ and by the linearity of the facilities,

$$
\begin{gathered}
E[Y]>\frac{\phi \tau w_{g}(\tau)+\phi(n-\tau) w_{f}(n-\tau)-n w_{f}(n)}{(n-1) d_{f}}= \\
\frac{\phi \tau w_{g}(\tau)+\phi(n-\tau)\left(w_{f}(n)+s d_{f}\right)-n w_{f}(n)}{(n-1) d_{f}} .
\end{gathered}
$$

Since $\pi_{\tau}$ is in equilibrium,

$$
R H S(46) \geq \frac{\phi \tau w_{f}(n-\tau+1)+(n(\phi-1)-\phi \tau) w_{f}(n)+\phi(n-\tau) \tau d_{f}}{(n-1) d_{f}} .
$$

Since $(n(\phi-1)-\phi \tau) w_{f}(n) \geq 0$,

$$
R H S(47) \geq \frac{\phi \tau\left(w_{f}(n-\tau+1)-w_{f}(n)\right)+\phi(n-\tau) \tau d_{f}}{(n-1) d_{f}}=
$$




$$
\frac{\phi \tau(\tau-1) d_{f}+\phi(n-\tau) \tau d_{f}}{(n-1) d_{f}}=\frac{\phi \tau(n-1)}{n-1}=\phi \tau .
$$

Therefore, if (44) does not hold,

$$
E[Y]>\phi \tau
$$

contradicting (45).

Case 2: $\tilde{p} \geq 1 / \phi$.

By (42) and since $\frac{w_{g}(1)-w_{f}(n)}{\tau\left(d_{f}+d_{g}\right)}=\tilde{p}$,

$$
E[Y] \leq \tilde{p} \tau+1
$$

Suppose in negation that the second constraint is satisfied, i.e., $R H S(43)=E[Y]\left(w_{f}(1)-\right.$ $\left.w_{f}(n)\right)+n w_{f}(n)>\beta$. Note that $\beta \geq \phi u(\tilde{\mathbf{q}})$. Therefore

$$
E[Y]>\frac{\phi u(\tilde{\mathbf{q}})}{\left(w_{f}(1)-w_{f}(n)\right)+n w_{f}(n)} .
$$

Hence, by part(ii) of Claim 3 and by linearity of the facilities

$$
\begin{gathered}
E[Y]>\frac{\phi n w_{f}(n)+\phi \tilde{p} d_{f}((n-\tau-1)(\tau+1)+(\tau+1) \tau)-n w_{f}(n)}{(n-1) d_{f}}= \\
\frac{n(\phi-1) w_{f}(n)+\phi \tilde{p} d_{f}(\tau+1)(n-1)}{(n-1) d_{f}} .
\end{gathered}
$$

Since $n(\phi-1) w_{f}(n) \geq 0$ and $\tilde{p} \leq 1 / \phi$

$$
R H S(50) \geq \tau+1 \text {. }
$$

Hence, $E[Y]>\tau+1$ contradicting (49) since $\tilde{p} \leq 1$.

This completes the proof of the theorem.

In Theorem 10 we derived, for every $n \geq 2$, the upper bound $\phi$ for the mediation value of the class of games, $\left(\left\{\Gamma_{F} \mid F \in \mathcal{Q N}_{n \times 2}\right.\right.$, all facilities of $\mathrm{F}$ are linear $\left.\}\right)$. When $n=2$, by Theorem 8 , we know that the mediation value equals 1 . Unfortunately, we do not know what is the mediation value for these classes of games when $n \geq 3$. However, the example below shows that, when $n=3$, the mediation value for this class is at least $\frac{9}{8}$; Hence, the mediation value is between 1.125 and $\phi \approx 1.618$.

Example 1 Let $n=3, M=\{f, g\}, w_{f}=(24,12,0)$, and $w_{g}=(8,8,8)$. It can be easily verified that $v_{N}(\Gamma)=32$, which is obtained, e.g., at the pure-strategy equilibrium in which two players choose $f$ and the other player chooses $g$. Consider the correlated strategy $\mu$, which assigns the probability $\frac{1}{6}$ to every strategy profile in which not all players choose the same facility. It is easily verified that $\mu$ is a correlated equilibrium and that the welfare at $\mu$ is 36 . Hence, the mediation value is at least $\frac{36}{32}$. 
For the next discussion it is useful to recall that the price of stability of a cost game is the ratio between the minimal cost at a mixed-strategy equilibrium and the minimal cost, and that the price of stability with utilities of a utility game is the ratio between the maximal welfare and the maximal welfare at a mixed-strategy equilibrium.

Let $n, m \geq 2$. Another open question for us is estimating the mediation value, of simple congestion games with $n$ players, $m$ facilities, and with nonnegative, non-increasing, and linear facilities. One can think that an upper bound for this class can be derived from the results about the price of stability for the analogous class of congestion games with costs. Indeed, Christodoulou and Koutsoupias (2005) proved that the price of stability of the class of congestion games with cost with $n$ players, $m$ facilities, and with nonnegative, linear, and non-decreasing cost functions is bounded above by 1.6. Had this result proven for the price of stability with utilities for our class of games it would have implied, in particular, that the mediation value of this class is bounded above by 1.6 , because the mediation value cannot exceed the price of stability with utilities. ${ }^{15}$ However, as discussed in the introduction, results about the price of stability in cost models cannot be transformed to results about the price of stability with utilities in utility models. To illustrate this, we show in the next example that the price of stability with utilities of the class $\left\{\Gamma_{F} \mid F \in\right.$ $\mathcal{Q N}_{2 \times 2}$, all facilities of $\mathrm{F}$ are linear $\}$ equals $\infty .^{16}$

Example 2 Let $x, \epsilon>0$ be fixed. Let $N=\{1,2\}, M=\{f, g\}$ and for every $d>\epsilon$, let $w_{f}^{d}=(x+d+\epsilon, x+\epsilon)$, let $w_{g}^{d}=(x, x)$, and $\Gamma^{d}$ be the associated congestion game. Since $f$ strictly dominates $g$, the strategy profile in which both players choose $f$ is the only mixed-equilibrium in the game $\Gamma^{d}$. The welfare obtained in this equilibrium is $2 x+2 \epsilon$. the strategy profile attaining the maximal welfare is the one in which each player chooses a different facility yielding a welfare of $2 x+\epsilon+d$. Since $\frac{2 x+\epsilon+d}{2 x+2 \epsilon} \rightarrow \infty$ when $d \rightarrow \infty$, the price of stability of $\left\{\Gamma_{F} \mid F \in \mathcal{Q N}_{2 \times 2}\right.$, all facilities of $\mathrm{F}$ are linear $\}$ equals $\infty$.

Theorems 9, and Example 1 show that correlation can be helpful in the context of (even non-increasing) congestion games. The next theorem shows that correlation can be helpful even in the narrow class of facility symmetric forms with nonnegative and non-increasing facilities:

Theorem $11 M V\left(\left\{\Gamma_{F} \mid F \in \mathcal{I N}_{n \times 2}\right\}\right)>1$ for every $n \geq 4$.

Proof: Let $n \geq 4$. It suffices to prove that there exists $F \in \mathcal{I N}_{n \times 2}$ such that $M V\left(\Gamma_{F}\right)>1$. Let $0<\epsilon<1$ be fixed and sufficiently small, and consider the following form, $F$ : $w_{1}=$ $w_{2}=(10 n, 1, \ldots, 1,1-\epsilon, 0)$. Note that the maximal welfare is obtained by any strategy profile in which exactly $n-1$ players choose the same facility, i.e., when the congestion vector is $\pi_{1}=(1, n-1)$ or $\pi_{2}=(n-1,1)$. Let $L$ be the set of all strategy profiles with a congestion vector $\pi_{1}$ or $\pi_{2}$. Note that there exist exactly $2 n$ strategy profiles in $L$. Let $\mu$ be the correlated strategy in which every strategy profile in $L$ is played with probability $\frac{1}{2 n}$. Since $\mu$ is a convex combination of welfare maximizers, the maximal welfare is attained at

15. Moreover, since $1.6 \leq \phi$, such a result would have saved us the tedious proof of Theorem 10 .

16. The proof of Christodoulou and Koutsoupias (2005) elegantly uses the fact that every congestion game has an exact potential. It is not known to us wether the potential approach could simplify our proof of Theorem 10. However, it turns out that their technique can not be applied directly to our setting because essentially they bound the total cost of the players on every facility separately in a given profile. 
$\mu$. We claim that $\mu$ is a correlated equilibrium. Indeed, by (2), it is easily verified that in $\Gamma_{F}$ there exists a unique constraint that should be satisfied in order to guarantee that $\mu$ is a correlated equilibrium. This constraint is: $\frac{10 n+((1-\epsilon)-1)(n-1)}{2 n} \geq 0$, which is indeed satisfied because we chose $\epsilon$ to be sufficiently small. Hence $\mu$ is a correlated equilibrium.

In order to prove that $M V\left(\Gamma_{F}\right)>1$, it suffices to prove that no mixed-strategy equilibrium obtains the maximal welfare. First, note that only strategy profiles in $L$ obtain the maximal welfare. Hence, if a pure-strategy equilibrium obtains the maximal welfare, it must belong to $L$. However, we claim that every strategy profile in $L$ is not in equilibrium; Indeed any player that chooses the facility chosen by $n-1$ players is better off deviating to the other facility since her utility will increase by $\epsilon$.

Therefore, it remains to show that in every mixed-strategy equilibrium, $\mathbf{p}=\left(p^{1}, \ldots, p^{n}\right)$, in which at least one player assigns a positive probability to each facility, there exists at least one pure strategy profile, $\mathbf{s}$, not in $L$, which is played with positive probability, that is $\mu_{\mathbf{p}}(\mathbf{s})>0$, where $\mu_{\mathbf{p}}$ is the correlated strategy associated with $\mathbf{p}$. Indeed, let $\mathbf{p}$ be a mixed-strategy equilibrium, and assume w.l.o.g. that player $i$ assigns a positive probability to both facilities, that is, $p^{i}(1), p^{i}(2)>0$. Assume in negation that $\mu_{\mathbf{p}}(\mathbf{t})>0$ implies $\mathbf{t} \in L$, and let $\mathbf{s}$ be a strategy profile for which $\mu_{\mathbf{p}}(\mathbf{s})>0$. Since $p^{i}(1), p^{i}(2)>0, \mu_{\mathbf{p}}\left(1, \mathbf{s}^{-i}\right)>0$ and $\mu_{\mathbf{p}}\left(2, \mathbf{s}^{-i}\right)>0$. Therefore, $\left(1, \mathbf{s}^{-i}\right) \in L$ and $\left(2, \mathbf{s}^{-i}\right) \in L$, which is impossible since $n \geq 4$.

If we further restrict the assumptions in Theorem 11 by requiring some concavity condition correlation cannot help anymore. We first define concavity:

Let $n \geq 2$. A function $v:\{1,2, \ldots, n\} \rightarrow \mathbb{R}^{+}$is concave if for every integer $k, 2 \leq k<n$, $v(k+1)-v(k) \leq v(k)-v(k-1)$.

Theorem 12 Let $n \geq m \geq 2$, and let $F \in \mathcal{I N}_{n \times m}$. Define $v(k)=k w(k)$ for every $1 \leq k \leq n$, where $w$ is the common facility payoff function, that is, $w=w_{j}$ for every $j \in M$. If $v$ is concave, there exists a pure-strategy equilibrium in $\Gamma_{F}$ which obtains the maximal welfare. Consequently, $M V\left(\Gamma_{F}\right)=1$.

Proof: For convenient we also define $v(0)=0$. We first define an operator on congestion vectors. Let $\pi=\left(\pi_{1}, \pi_{2}, \ldots, \pi_{m}\right)$ be a congestion vector and let $j, l \in M$ be any pair of distinct facilities. Let $\pi^{*}[j, l]$ be the congestion vector obtained from $\pi$ by replacing $\pi_{j}$ and $\pi_{l}$ with $\pi_{j}^{*}=\left\lceil\frac{\pi_{j}+\pi_{l}}{2}\right\rceil$ and $\pi_{l}^{*}=\left\lfloor\frac{\pi_{j}+\pi_{l}}{2}\right\rfloor$, respectively, where $\lfloor x\rfloor(\lceil x\rceil)$ denotes the largest (least) integer that is not higher (lower) than $x$. Observe that $u(\pi)=\sum_{i=1}^{m} v\left(\pi_{i}\right)$, and therefore, by the concavity of $v$,

$$
u\left(\pi^{*}[j, l]\right) \geq u(\pi)
$$

Next we describe a finite sequence of congestion vectors, such that each one of them obtains the maximal welfare and the last one is also in equilibrium. Before that, set $k_{1}=$ $\left\lfloor\frac{n}{m}\right\rfloor, k_{2}=\left\lceil\frac{n}{m}\right\rceil$, and note that every congestion vector in which each coordinate is either $k_{1}$ or $k_{2}$ is in equilibrium.

Pick any congestion vector $\pi$ that obtains the maximal welfare. If $\pi$ is in equilibrium, we are done. Otherwise, not all of its coordinates are $k_{1}$ or $k_{2}$. In particular, there exist two distinct coordinates $\hat{j}$ and $\hat{l}$ such that $\pi_{\hat{j}} \leq k_{1}, \pi_{\hat{l}} \geq k_{2}$, and at least one inequality is strict. Construct $\pi^{*}=\pi^{*}[\hat{j}, \hat{l}]$. By $(51), u\left(\pi^{*}\right) \geq u(\pi)$, and therefore, $\pi^{*}$ obtains the maximal welfare. If $\pi^{*}$ is in equilibrium we are done. Otherwise let $\pi=\pi^{*}$ and repeat the 
process. If the sequence of $\pi^{*}$ 's does not terminate, it eventually reaches a $\pi^{*}$ in which every coordinates equals $k_{1}$ or $k_{2}$, i.e., it reaches an equilibrium -a contradiction. Therefore, the sequence terminates after finite number of stages, and hence the last congestion vector in the sequence is in equilibrium and attains the maximal welfare.

\subsection{The Enforcement Value}

We already know from the construction in the proof of Theorem 4 that the enforcement value of the class $D=\left\{\Gamma_{x} \mid x>1\right\}$ of Prisoner's Dilemma games described in Figre 5 is unbounded. It is easily verified that every game in this class is a potential game, and therefore, by (Monderer \& Shapley, 1996), it is a congestion game. Moreover, it can be easily verified that each such game can be derived from a simple and nonnegative congestion form in $\mathcal{Q}_{2 \times 2}$. Therefore, $E V\left(\left\{\Gamma_{F} \mid F \in \mathcal{Q}_{2 \times 2}\right)=\infty\right.$. However, as noticed by Monderer (2007), not every congestion game with nonnegative utilities can be represented by a congestion form with nonnegative and non-increasing facilities. In particular, it can be shown that the Prisoner's Dilemma games in $D$ cannot be represented by simple congestion forms with nonnegative and non-increasing facilities. The next theorem shows that even if the facility payoff functions are restricted to be non-increasing, the enforcement value remains unbounded for two player games.

Theorem $13 E V\left(\left\{\Gamma_{F} \mid F \in \mathcal{Q N}_{2 \times m}\right\}\right)=\infty$ for every $m \geq 2$.

Proof: Consider the games $\Gamma^{d}, d>0$, given in Example 2. Since using facility $f$ strictly dominates using $g$, by Lemma 1, the strategy profile in which both players choose $f$ is the unique correlated equilibrium in $\Gamma^{d}$. As is proved in Example $2, \frac{o p t\left(\Gamma^{d}\right)}{v_{C}\left(\Gamma^{d}\right)} \rightarrow \infty$, when $d \rightarrow \infty$. This proves the theorem for $m=2$. For $m>2$ the proof is obtained, as above, by naturally modifying the games in Example 2.

Since Theorem 13 deals with two players, we deduce that the enforcement value is unbounded also for the class of games generated by linear facilities in $\mathcal{Q N}_{2 \times m}$ because for two players, every non-increasing facility is linear.

Note that the proof of Theorem 13 utilizes games, which posses strictly, and in particular, weakly dominant strategies. The next theorem deals with games without weakly dominant strategies.

\section{Theorem 14}

(i) $E V\left(\left\{\Gamma_{F} \mid F \in \mathcal{Q N}_{2 \times 2}\right.\right.$, there are no weakly dominant strategies $\left.\}\right)=1$.

(ii) $E V\left(\left\{\Gamma_{F} \mid F \in \mathcal{Q N}_{3 \times 2}\right.\right.$, there are no weakly dominant strategies $\left.\}\right)=\infty$.

Proof: Assertion (i) follows from Theorem 8. As for Assertion (ii), the proof follows by observing that the game in the proof of Theorem 5 is a simple congestion game with nonincreasing facilities.

The next theorem shows that the enforcement value tends to $\infty$ when the number of players tends to $\infty$ even when restricting the facilities to be symmetric and non-increasing.

Theorem $15 \lim _{n \rightarrow \infty} E V\left(\left\{\Gamma_{F} \mid F \in \mathcal{I N}_{n \times 2}\right\}\right)=\infty$. 
Proof: Consider the following family of forms $F_{n} \in \mathcal{I N}_{n \times 2}, n \geq 3: M=\{f, g\}$ and let $w_{f}=w_{g}=(\sqrt{n}, 1,0,0, . ., 0)$. Observe that the congestion vector, $\pi_{n}=(1, n-1)$, obtains the maximal welfare, which equals $\sqrt{n}$. Therefore, in order to prove the theorem, It suffices to show that

$$
v_{C}\left(\Gamma_{F_{n}}\right) \leq 3 \text { for every } n \geq 3 .
$$

Let then $n \geq 3$ be fixed. In order to prove (52), we use the dual program $(\widehat{D})$. By the weak duality theorem, every feasible solution of the dual problem satisfies $\beta \geq v_{C}\left(\Gamma_{F_{n}}\right)$. Therefore, it suffices to prove that the dual problem has a feasible solution with $\beta=3$. However, in our case, the dual problem is just the problem, $\widehat{D}_{\Gamma}$ described right after (40). We will find a feasible solution to $\widehat{D}_{\Gamma}$, in which all dual variables except $\beta$ have an identical value $x$, i.e., $x=\alpha^{i}(f \mid g)=\alpha^{i}(g \mid f)$ for each player $i$. Under this restriction, the dual program, $\widehat{D}_{\Gamma}$ reduces to:

$$
\left\{\begin{array}{l}
\min \beta \\
\text { s.t. } \\
x \geq 0 \\
(-\sqrt{n}+n-1) x+\beta \geq \sqrt{n}, \\
-2 x+\beta \geq 1, \\
n \sqrt{n}+\beta \geq 0,
\end{array}\right.
$$

which has the feasible solution, $(x, \beta)=(1,3)$. Therefore $(52)$ holds.

Although the enforcement value may be unbounded when we have facility symmetric congestion forms, it is interesting to characterize those congestion games for which correlation enables to get the maximal welfare. This is done in the next theorem, but first we need the following notations. Let $F$ be a congestion form, and Let $\pi$ be a congestion vector in $F$. Let $\tau: M \rightarrow M$ be a one to one function, i.e., $\tau$ is a permutation of the set of facilities. We define the congestion vector $\tau \pi=(\tau \pi)_{j \in M}$ as follows: $(\tau \pi)_{j}=\pi_{\tau(j)}$ for every facility $j$. Recall that $B_{\pi}$ is the set of all strategy profiles that induce the congestion vector $\pi$. We further define, $L_{\pi}=\bigcup_{\tau} B_{\tau \pi}$ to be the set of all strategy profiles that induce a permutation of the congestion vector $\pi$.

Let $F \in \mathcal{I}_{n \times m}$, that is, all facilities in $F$ are symmetric. Therefore, $u(\pi)=u(\tau \pi)$ for every permutation $\tau$, and, in addition, for every pair of strategy profiles $A, B \in L_{\pi}$, $u(A)=u(B)$.

Theorem 16 Let $n, m \geq 2$, and let $F \in \mathcal{I}_{n \times m}$. Then, $v_{C}\left(\Gamma_{F}\right)=\operatorname{opt}\left(\Gamma_{F}\right)$ if and only if there exist a congestion vector $\pi=\left(\pi_{1}, \ldots, \pi_{m}\right)$ and a correlated equilibrium $\mu \in C\left(\Gamma_{F}\right)$ such that the following two conditions hold:

1. $u(\pi)=\operatorname{opt}\left(\Gamma_{F}\right)$.

2. $\mu$ is distributed uniformly over all strategy profiles in $L_{\pi}$; that is, $\mu(\mathbf{d})=\mu(\overline{\mathbf{d}})$ for every $\mathbf{d}, \overline{\mathbf{d}} \in L_{\pi}$, and $\mu(\mathbf{d})=0$ for every $\mathbf{d} \notin L_{\pi}$.

The following lemma (Schrijver, 1986, page 61) is used in our proof: 
Lemma 6 (Variant of the Farkas Lemma) Let $s, t$ be positive integers. Given a matrix $A$ of dimensions $s \times t$ and a vector $\mathbf{b} \in \mathbb{R}^{s}$, one and only one of the following systems has a solution:

(i) $\quad \mathbf{A x}=\mathbf{b}, \quad \mathbf{x} \geq 0, \quad \mathbf{x} \in \mathbb{R}^{t} ;$

(ii) $\quad \mathbf{y}^{T} \mathbf{A} \geq 0, \quad \mathbf{y}^{T} \mathbf{b}<0, \quad \mathbf{y} \in \mathbb{R}^{s}$.

\section{Proof of Theorem 16: ${ }^{17}$}

Clearly, if there exist a congestion vector $\pi$ and a correlated equilibrium $\mu \in C\left(\Gamma_{F}\right)$, which satisfy both conditions, $v_{C}\left(\Gamma_{F}\right)=\operatorname{opt}\left(\Gamma_{F}\right)$. Before we prove the other direction we need Claim 1 below.

Let $w$ be the common facility payoff function, that is, $w=w_{j}$ for every $j \in M$. For every congestion vector $\pi$ we define $Z(\pi)$ as follows:

$$
Z(\pi)=\frac{\sum_{j=1}^{m} \pi_{j} \sum_{k \neq j}\left(w\left(\pi_{j}\right)-w\left(\pi_{k}+1\right)\right)}{m ! n} .
$$

Claim 1: Suppose $v_{C}\left(\Gamma_{F}\right)=\operatorname{opt}\left(\Gamma_{F}\right)$. Let $\pi=\left(\pi_{1}, \ldots, \pi_{m}\right)$ be a congestion vector in which $u(\pi)=\operatorname{opt}\left(\Gamma_{F}\right)$, and let $\mu$ be a correlated strategy distributed uniformly over all elements in $L_{\pi} \cdot \mu$ is a correlated equilibrium if and only if $Z(\pi) \geq 0$.

Proof: Let $D_{\pi, i, j}$ be the set of all strategy profiles in which player $i$ chooses facility $j$ and the congestion vector is $\pi$. That is $D_{\pi, i, j}=\left\{\mathbf{d}: \mathbf{d} \in B_{\pi} \quad, \mathbf{d}^{i}=j\right\}$. $i \in N$.

Let $s_{\pi}(j)=\left(\begin{array}{c}n-1 \\ \pi_{j}-1\end{array}\right) \prod_{l=1}^{m}\left(\begin{array}{c}n-\pi_{j}-\sum_{k=1}^{l-1} \pi_{k} 1_{k \neq j} \\ \pi_{l} 1_{l \neq j}\end{array}\right)$, and note that $\left|D_{\pi, i, j}\right|=s_{\pi}(j)$ for every

Since $\mu$ is distributed uniformly over all elements in $L_{\pi}, \mu$ is a correlated equilibrium if and only if

$$
\frac{\sum_{j=1}^{m} s_{\pi}(j) \sum_{k \neq j}\left(w\left(\pi_{j}\right)-w\left(\pi_{k}+1\right)\right)}{\left|L_{\pi}\right|} \geq 0 .
$$

Hence, it suffices to show that $\operatorname{LHS}(54)=Z(\pi)$. This follows since $\left|L_{\pi}\right|=\left|B_{\pi}\right| m !=$ $\left(\begin{array}{c}n \\ \pi_{1}\end{array}\right)\left(\begin{array}{c}n-\pi_{1} \\ \pi_{2}\end{array}\right) \cdots\left(\begin{array}{c}n-\sum_{j=1}^{m-2} \pi_{j} \\ \pi_{m-1}\end{array}\right) m$ ! and $\frac{\left(\begin{array}{c}n \\ k-1\end{array}\right)}{\left(\begin{array}{c}n \\ k\end{array}\right)}=\frac{k}{n}$

We proceed with proving the remaining direction. Suppose that $v_{C}\left(\Gamma_{F}\right)=\operatorname{opt}\left(\Gamma_{F}\right)$, and assume for a contradiction that there do not exist a congestion vector $\pi$ and a correlated equilibrium $\mu \in C\left(\Gamma_{F}\right)$ such that $\mu$ is distributed uniformly over all elements in $L_{\pi}$ and $u(\pi)=\operatorname{opt}\left(\Gamma_{F}\right)$. Recall that for every strategy profile $\mathbf{d} \in \mathbf{S}, \pi^{\mathbf{d}}$ is the congestion vector induced by $\mathbf{d}$ and that $\pi_{k}^{\mathbf{d}}$ is the number of players that choose facility $k$ in $\mathbf{d}$. Let $D=$ $\bigcup_{\left\{\mathbf{d}: u\left(\pi^{d}\right)=o p t\left(\Gamma_{F}\right)\right\}} L_{\pi^{\mathrm{d}}}$. Because of our negation assumption, Claim 1 implies that

$$
Z\left(\pi^{\mathbf{d}}\right)<0 \text { for every } \mathbf{d} \in D .
$$

We are about to utilize Lemma 6 , and for that matter set $J=|D|$. Define the matrix $A$ of size $J \times n\left(m^{2}-m\right)$ as follows:

$$
A(\mathbf{d}, i j k)=\frac{\left[w\left(\pi_{j}^{\mathbf{d}}\right)-w\left(\pi_{k}^{\mathbf{d}}+1\right)\right] 1_{i j}(\mathbf{d})}{m ! n},
$$

17. The proof technique was inspired by Nau and McCardle (1990). 
where each row corresponds to a strategy profile $\mathbf{d} \in D$ and column $i j k$ corresponds to player $i$ who chooses $j t h$ strategy (facility) and deviates to the $k t h$ strategy $(j \neq k)$, and:

$$
1_{i j}(\mathbf{d})= \begin{cases}1 & d^{i}=j \\ 0 & \text { otherwise. }\end{cases}
$$

Every row $\mathbf{d}$ in the matrix $A$ corresponds to strategy profile $\mathbf{d} \in D$, which in turn, corresponds to a congestion vector $\pi^{\mathbf{d}}=\left(\pi_{1}^{\mathbf{d}}, \ldots, \pi_{m}^{\mathbf{d}}\right)$. Set $b(\mathbf{d})=Z\left(\pi^{\mathbf{d}}\right)$ for every $\mathbf{d} \in$ $D$. Note that the column vector $\mathbf{x}=(1,1, \ldots, 1)^{T} \in \mathbb{R}^{J}$ satisfies $(i)$ in Lemma 6 , where $\mathbf{b}=(b(\mathbf{d}))_{\mathbf{d} \in D}$. Therefore, by Lemma 6 , system $(i i)$ in Lemma 6 does not have a solution. Because $v_{C}\left(\Gamma_{F}\right)=\operatorname{opt}\left(\Gamma_{F}\right)$, there exists a correlated equilibrium, say $\bar{\mu}$, satisfying $u(\bar{\mu})=$ $v_{C}\left(\Gamma_{F}\right)=\operatorname{opt}\left(\Gamma_{F}\right)$. Obviously, $\bar{\mu}$ is supported in $D$, that is $\mu(\mathbf{d})=0$ for every $\mathbf{d} \notin D$. Let $\mathbf{y}(\mathbf{d})=\bar{\mu}(\mathbf{d})$ for every $\mathbf{d} \in D$, and let $\mathbf{y}=(y(\mathbf{d}))_{\mathbf{d} \in D}$. Since $\bar{\mu}$ is a correlated equilibrium concentrated on $D, \mathbf{y}^{T} A \geq 0$, and since $\mathbf{y}$ does not satisfy (ii),

$$
\mathbf{y}^{T} \mathbf{b} \geq 0
$$

On the other hand, since $\mathbf{y}$ is a probability distribution on $D$, and (55) holds,

$$
\mathbf{y}^{T} \mathbf{b}=\sum_{\mathbf{d} \in D} y(\mathbf{d}) b(\mathbf{d})=\sum_{\mathbf{d} \in D} \bar{\mu}(\mathbf{d}) b(\mathbf{d})<0
$$

contradicting (57). Therefore, our negation assumption cannot hold, and the theorem is proved.

Theorem 16 shows that for symmetric congestion games, under the conditions of the theorem, correlation helps in obtaining the maximal welfare. The next example shows that there exist such games in which mixed-strategy equilibrium is not as useful as correlated equilibrium:

Example 3 Let $F \in \mathcal{I}_{6 \times 2}$. Let $w_{j}=(1.5,1,4,4.5,4.5,3)$ for every $j=1,2$. It is easy to verify that the maximal welfare is obtained in a strategy profile $\mathbf{A}$ if and only if $\mathbf{A} \in$ $L_{\pi_{1}} \cup L_{\pi_{2}}$, where $\pi_{1}=(3,3)$ and $\pi_{2}=(1,5)$. Let $\mu$ be the correlated strategy, which is distributed uniformly over $L_{\pi_{2}}$. It can be checked that $\mu$ is a correlated equilibrium. Hence, by Theorem 16, $v_{C}\left(\Gamma_{F}\right)=\operatorname{opt}\left(\Gamma_{F}\right)$. On the other hand, note that both $\pi_{1}$ and $\pi_{2}$ are not in equilibrium. Hence, in every mixed-strategy profile, a profile which does not obtain the maximal welfare will be played with positive probability. Therefore the mediation value is greater than 1, i.e. the best mixed-strategy equilibrium is less useful than the best correlated equilibrium.

\section{Conclusion}

In this work we have introduced and studied two measures for the value of correlation in strategic interactions: the mediation value and the enforcement value. These measures complement existing measures appearing in the price of anarchy literature, which are comparing the maximal welfare (when agent behavior can be dictated) to the welfare obtained in Nash equilibrium (when agents are selfish). Indeed, correlation captures many interesting situations, which are common to computing systems and e-commerce applications. In 
many systems a reliable party can advise the agents on how to behave but can not enforce such behavior. The gain that may be obtained by this capability is the major subject of the study presented in this work. We studied and showed the power of this approach, both for general games and in the context of congestion games.

\section{Acknowledgments}

A preliminary version of this paper appears in the proceedings of the 20th conference on Uncertainty in Artificial Intelligence (UAI-05). We thank the German-Israeli Foundation (GIF) for a financial support

\section{References}

Anshelevich, E., Dasgupta, A., Kleinberg, J., Tardos, E., Wexler, T., \& Roughgarden, T. (2004). The Price of Stability for Network Design with Fair Cost Allocation. In Proceedings of the 45th IEEE Symposium on Foundations of Computer Science, (FOCS-04), pp. 59-73.

Ashlagi, I., Monderer, D., \& Tennenholtz, M. (2008). Mediators in position auctions. To appear in Games and Economic Behavior. A shorter version appears in the Proceedings of the 8th ACM conference on Electronic Eommerce.

Aumann, R. (1974). Subjectivity and Correlation in Randomized Strategies. Journal of Mathematical Economics, 1, 67-96.

Aumann, R. (1987). Correlated Equilibrium as an Expression of Bayesian Rationality. Econometrica, 55, 1-18.

Christodoulou, G., \& Koutsoupias, E. (2005). On the Price of Anarchy and Stability of Correlated Equilibria of Linear Congestion Games. In Proceedings of the 13th Annual European Symposium, ESA 2005, pp. 59-70.

Czumaj, A., \& Vocking, B. (2002). Tight Bounds For Worst Case Equilibria. In Proceedings of the 13th Annual Symposium on Discrete Algorithms, pp. 413-420.

Hart, S., \& Schmeidler, D. (1989). Existence of Correlated Equilibria. Math. Oper. Res., $14,18-25$.

Kakade, S., Kearns, M., Langford, J., \& Ortiz, L. (2003). Correlated equilibria in graphical games. In Proceedings of the 4th ACM conference on Electronic commerce, pp. 42-47.

Koutsoupias, E., \& Papadimitriou, C. (1999). Worst-Case Equilibria. In Proceedings of the 16th Annual Symposium on Theoretical Aspects of Computer Science, pp. 404-413.

Marvonicolas, M., \& Spirakis, P. (2001). The Price of Selfish Routing. In Proceedings of the 33rd Symposium on Theory of Computing, pp. 510-519.

Monderer, D. (2007). Multipotential Games. In Twentieth International joint conference on Artificial Intelligence (IJCAI-0\%) . 
Monderer, D., \& Shapley, L. (1996). Potential Games. Games and Economic Behavior, 14, $124-143$.

Monderer, D., \& Tennenholtz, M. (2004). K-Implementation. Journal of Artificial Intelligence Research (JAIR), 21, 37-62.

Monderer, D., \& Tennenholtz, M. (2006). Strong mediated equilibrium. In Proceedings of the AAAI.

Nash, J. (1951). Noncooperative Games. Ann. Math., 54, 286-295.

Nau, R. F., \& McCardle, K. F. (1990). Coherent Behavior in Noncooperative Games. Journal of Economic Theory, 50, 424-444.

Papadimitriou, C. (2001). Algorithms, Games, and the Internet. In Proceedings of the 16th Annual ACM Symposium on Theoretical Aspects of Computer Science, pp. 749-753.

Papadimitriou, C. (2005). Computing correlated equilibria in multi-player games. In Proceedings of the thirty-seventh annual ACM symposium on Theory of computing, pp. $49-56$.

Peeters, R., \& Potters, J. (1999). On the Structure of the Set of Correlated Equilibria in Two-by-Two Bimatrix Games. Technical report, Tilburg - Center for Economic Research.

Rosenthal, R. (1973). A Class of Games Possessing Pure-Strategy Nash Equilibria. International Journal of Game Theory, 2, 65-67.

Roughgarden, T. (2002). Selfish Routing. PhD Thesis, Cornell University.

Roughgarden, T., \& Tardos, E. (2002). How Bad is Selfish Routing?. Journal of the ACM, 49(2), 236-259.

Rozenfeld, O., \& Tennenholtz, M. (2007). Routing mediators. In Proceedings of the 23rd International Joint Conferences on Artificial Intelligence(IJCAI-0\%), pp. 1488-1493.

Schrijver, A. (1986). Theory of Linear and Integer Programming. Wiley, New York.

Shoham, Y., \& Tennenholtz, M. (1995a). Artificial Social Systems. Computers and Artificial Intelligence, 14, 533-562.

Shoham, Y., \& Tennenholtz, M. (1995b). On Social Laws for Artificial Agent Societies: Off-Line Design. Artificial Intelligence, 73, 231-252. 